\title{
Increasing the throughput of sensitive proteomics by plexDIA
}

\author{
Jason Derks, ${ }^{1,2,3}$ Andrew Leduc, ${ }^{1,2,3}$ R. Gray Huffman, ${ }^{1,2,3}$ Harrison Specht, ${ }^{1,2,3}$ \\ Markus Ralser, ${ }^{4,5}$ Vadim Demichev ${ }^{4, \bowtie}$ \& Nikolai Slavov ${ }^{1,2,3, \varpi}$ \\ ${ }^{1}$ Department of Bioengineering, Northeastern University, Boston, MA 02115, USA \\ ${ }^{2}$ Barnett Institute, Northeastern University, Boston, MA 02115, USA \\ ${ }^{3}$ Department of Chemistry and Chemical Biology, Northeastern University, Boston, MA 02115, USA \\ ${ }^{4}$ Charité - Universitätsmedizin Berlin, Germany \\ ${ }^{5}$ Molecular Biology of Metabolism Laboratory, The Francis Crick Institute, London, UK \\ $\bowtie$ Correspondence: nslavov@northeastern.edu and vadim.demichev@charite.de \\ $\in$ Data, code \& protocols: plexDIA.slavovlab.net
}

Current mass-spectrometry methods enable high-throughput proteomics of large sample amounts, but proteomics of low sample amounts remains limited in depth and throughput. We aimed to increase the throughput of high-sensitivity proteomics while achieving high proteome coverage and quantitative accuracy. We developed a general experimental and computational framework, plexDIA, for simultaneously multiplexing the analysis of both peptides and samples. Multiplexed analysis with plexDIA increases throughput multiplicatively with the number of labels without reducing proteome coverage or quantitative accuracy. Specifically, plexDIA using 3-plex nonisobaric mass tags enables quantifying 3-fold more protein ratios among nanogram-level samples. Using 1 hour active gradients and first-generation $Q$ Exactive, plexDIA quantified about 8,000 proteins in each sample of labeled 3-plex sets. Furthermore, plexDIA increases the consistency of protein quantification, resulting in over 2 -fold reduction of missing data across samples. We applied plexDIA to quantify proteome dynamics during the cell division cycle in cells isolated based on their DNA content. The high sensitivity and accuracy of plexDIA detected many classical cell cycle proteins and discovered new ones. These results establish a general framework for increasing the throughput of highly sensitive and quantitative protein analysis. 


\section{Introduction}

Mass-spectrometry (MS) methods can achieve deep proteome coverage ${ }^{1,2}$, low missing data ${ }^{3}$, high throughput ${ }^{4,5}$, and high sensitivity ${ }^{6}$. However, simultaneously achieving all these objectives is an outstanding challenge ${ }^{7,8}$. Resolving this challenge will empower biomedical projects that are impractical with current methods ${ }^{8,9}$. Towards this goal, the throughput of sensitive protein analysis may be increased by different strategies: (i) increasing sample throughput and robustness by chemical labeling, and (ii) decreasing MS analysis time per sample by simultaneous (parallel) analysis of multiple peptides. These strategies are complementary, and we sought to combine them to achieve a multiplicative increase in the rate of quantifying the proteomes of limited sample amounts.

Chemical labeling is often used with data-dependent acquisition (DDA) to increase throughput and control for shared artifacts, such as disturbances in peptide separation and ionization ${ }^{10-12}$. Since quantifying a mammalian proteome requires analyzing hundreds of thousands of precursor ions and DDA methods analyze one precursor per MS2 scan, even the most optimized DDA methods require up to a day of LC-MS/MS for deep proteome analysis ${ }^{1}$. Nonisobaric labels, such as mTRAQ and dimethyl labeling allow for sample multiplexing but further increase the number of precursor ions and thus the time needed for MS1-multiplexed DDA analysis ${ }^{13}$. In contrast, approaches using isobaric labels (such as TMT; tandem mass tags) do not increase the number of distinguishable precursor ions and can reduce the analysis time per sample ${ }^{14,15}$, albeit quantification with TMT is often significantly affected by coisolation interference ${ }^{11,16}$.

The throughput of DDA analysis can be further increased by decreasing the ion accumulation times for MS2 scans, though this throughput increase reduces sensitivity ${ }^{7}$. Indeed, sensitive analysis of small sample amounts requires long ion accumulation times ${ }^{6,17,18}$. Even with short ion accumulation times for unlimited sample amounts, the requirement to serially analyze hundreds of thousands of precursor ions remains a major challenge for simultaneously achieving high throughput and deep proteome coverage by serial precursor analysis.

A fundamental solution to this challenge is the concept of isolating and analyzing multiple precursor ions simultaneously by data-independent acquisition (DIA) ${ }^{19}$. This concept has been implemented into powerful methods for label-free DIA (LF-DIA) protein analysis ${ }^{20-24}$. Such par- 
allel analysis of peptides decreases the time needed to analyze thousands of precursor ions and makes the throughput of optimized LF-DIA and TMT-DDA workflows comparable, allowing routine quantification of about 6,000 proteins in 2 hours $^{15}$. Recent DIA technologies further enabled quantification of over 8,000 proteins in 1.5 hours $^{25}$ and TMTpro tags increased multiplexing to 18plex for DDA methods ${ }^{4}$. Thus multiplexed DDA and LF-DIA afford comparable throughput.

We sought to further increase the throughput of sensitive DIA by multiplexing samples labeled with nonisobaric isotopologous mass tags, capitalizing on the fact that increasing the number of precursor ions does not increase the time needed to analyze them via tandem DIA-MS, in contrast to DDA-MS ${ }^{13,19}$. This creates a hypothetical possibility that we sought to test: The number of protein ratios accurately quantified by multiplexed DIA may increase multiplicatively with the number of labels used. If feasible, this possibility may enable higher throughput and more sensitive multiplexed proteomics, including single-cell proteomics as previously suggested ${ }^{7,26}$. While the feasibility of DIA multiplexed by SILAC ${ }^{27}$ or pulsed SILAC ${ }^{28,29}$ has been clearly demonstrated, its ability to multiplicatively increase quantitative data points remains unclear. Similarly, clever strategies have used both isobaric and isotopologous tags to multiplex DIA, but they have afforded the quantification of relatively few proteins ${ }^{30-32}$. Thus, the potential of multiplexed DIA to increase sample throughput while preserving proteome coverage and quantification accuracy has not been realized due to the increased complexity of DIA data from labeled samples ${ }^{31-35}$.

We hypothesized that an optimized experimental and analytical framework may enable $n$-fold multiplexed DIA to increase $n$-fold the number of accurate protein data points. We test this hypothesis for $n=3$ using amine-reactive nonisobaric isotopologous mass tags (mTRAQ), hoping that this particular choice of mass tags can establish a framework that will in the future generalize to a variety of isotopologous non-isobaric mass tags with higher capacity for multiplexing. Specifically, we sought to develop a general framework and an analysis pipeline to increase the throughput of sensitive and quantitative protein analysis via plexDIA. 


\section{Results}

\section{plexDIA benchmarks}

We sought to evaluate whether plexDIA can multiplicatively increase the number of quantitative data points relative to matched label-free DIA (LF-DIA) analysis while maintaining comparable quantitative accuracy. Towards that goal, we mixed proteomes in precisely specified ratios shown in Fig. 1a, thus creating a benchmark of known protein ratios for thousands of proteins spanning a wide dynamic range of abundances, similar to previous benchmarks ${ }^{21}$. Specifically, we made three samples (A, B, and C), each with an exactly specified amount of E. coli, S. cerevisiae, and $H$. sapiens (U-937 and Jurkat) cell lysate, Fig. 1a. A distinct aspect of this design is the incorporation of human proteomes of different cell types, which affords additional benchmarking for the reproducibility of protein identification across diverse samples and for relative protein quantification.

Each sample was either analyzed by label-free DIA (LF-DIA) or labeled with one of three amine-reactive isotopologous chemical labels (mTRAQ: $\Delta 0, \Delta 4$, or $\Delta 8$ ), Fig. 1a. With this experimental design, plexDIA enables 3-fold reduction in LC-MS/MS time per sample, which provides nearly 3-fold reduction in the overall cost per sample because most of the cost stems from LCMS/MS fees while the cost of labeling is low, Fig. 1b. The combined labelled samples were analyzed by plexDIA, and the result was used to benchmark proteomic coverage, quantitative accuracy, precision, and reproducibility across runs relative to LF-DIA of the same samples. LF-DIA and plexDIA were evaluated with two data acquisition methods, V1 and V2, shown in Fig. 1c. V1 included multiple high-resolution MS1 survey scans to increase the temporal resolution of precursor sampling as previously reported ${ }^{3}$ while V2 included more MS2 scans to increase proteome coverage, Fig. 1c; The only difference between the duty cycles of LF-DIA and plexDIA was a $100 \mathrm{~m} / \mathrm{z}$ increment in the MS1 and MS2 windows of plexDIA to account for the mass of mTRAQ added to the peptides; see methods.

\section{plexDIA increases throughput}

To directly benchmark the analysis of $500 \mathrm{ng}$ protein samples by plexDIA relative to LF-DIA, the multiplexed and label-free samples described in Fig. 1 were analyzed in triplicate by LC-MS/MS 


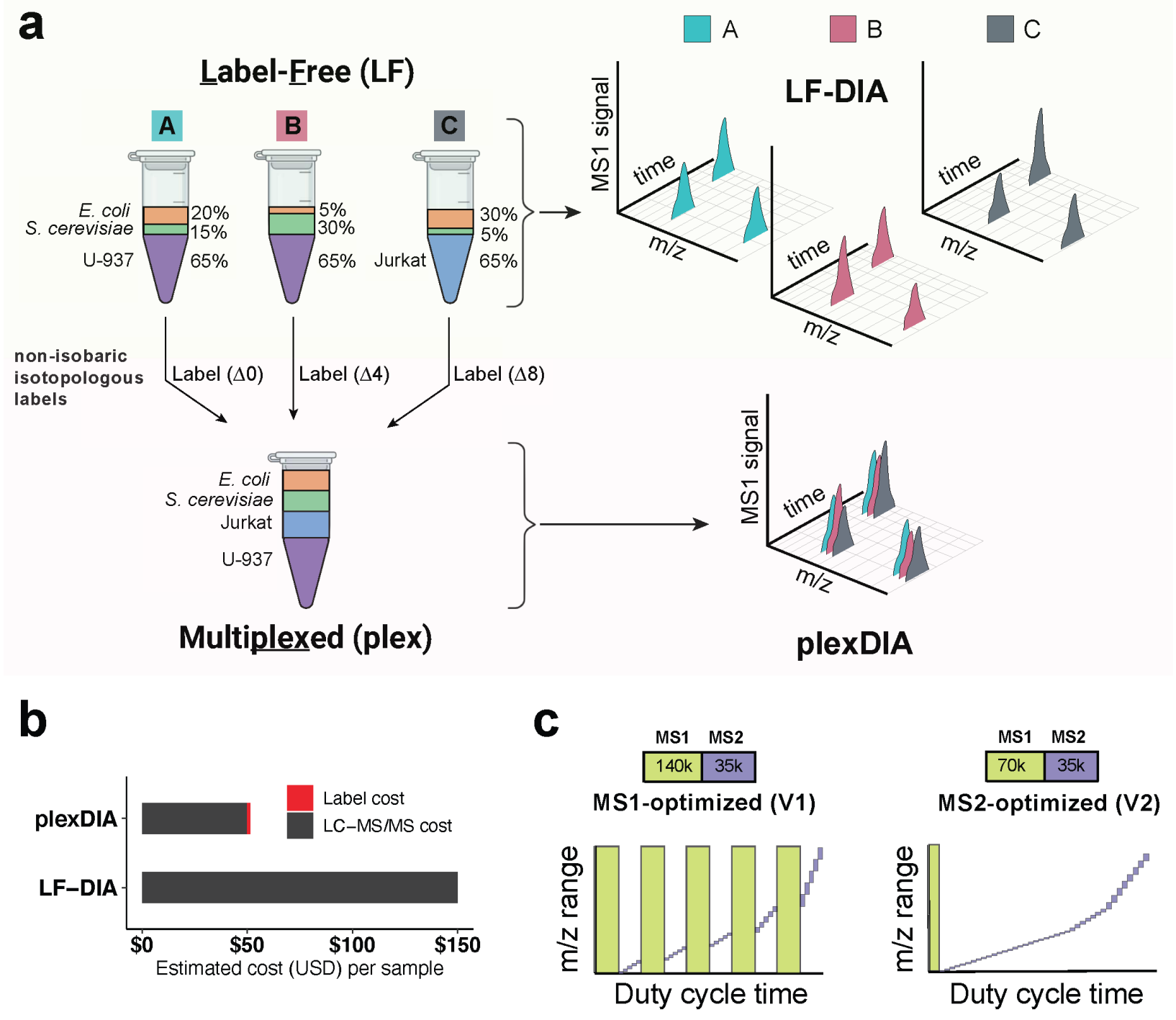

Figure 1 | Experimental design for acquisition and evaluation of plexDIA data. (a) Standards used for benchmarking LF-DIA and plexDIA quantification were prepared by mixing the proteomes of different species and cell types as shown. LF-DIA analyzed 500ng from each of the 3 samples (A, B, C) separately, while plexDIA analyzed a mixture of these samples labeled with nonisobaric mass tags (mTRAQ). (b) Analyzing samples by plexDIA is cheaper than analysis by LF-DIA because running $n$ samples in parallel reduces the LC-MS/MS time per sample $n$-fold and the cost of labeling is low. This estimate is based on a facility fee of 150 USD / hour of active gradient. (c) We benchmarked the performance of plexDIA with two DIA methods: V1 is an MS1-optimized method that utilizes frequent, high resolution MS1 scans to facilitate accurate quantification while V2 is an MS2-optimized method which takes a single MS1 scan and more MS2 scans per duty cycle. 
on Thermo Q-Exactive (first generation) with a 60-min active nano-LC gradient. The throughput increases for duty cycles V1 (Fig. 2) and V2 (Fig. S1) were very similar, except that V2 achieved larger proteome coverage, detecting 266,723 precursors per plexDIA run. This corresponds to 7,972 proteins groups per sample, of which 7,359 were quantified in all 3 samples. The parallel data acquisition by all DIA methods resulted in a larger number of identified peptides and proteins compared to the DDA runs, Fig. 2a,b. Both V1 and V2 resulted in almost 3-fold more data points for plexDIA compared to LF-DIA. Below, we compare the coverage more rigorously, focusing on the number of protein ratios that can be estimated and on their accuracy.

a

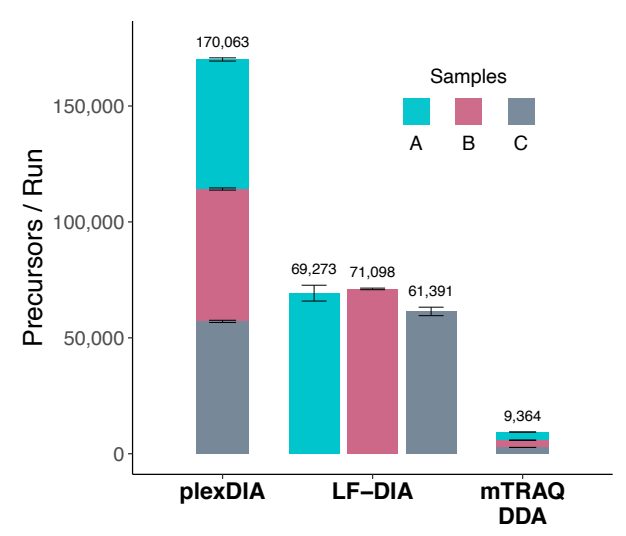

C

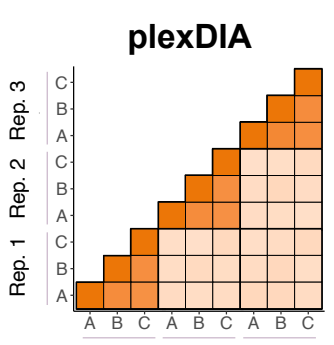

Rep. 1 Rep. 2 Rep. 3

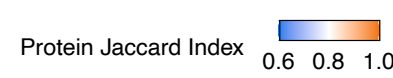

LF-DIA

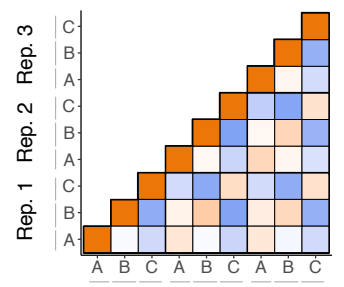

$\begin{array}{lll}\text { Rep. } 1 & \text { Rep. } 2 \text { Rep. } 3\end{array}$ b
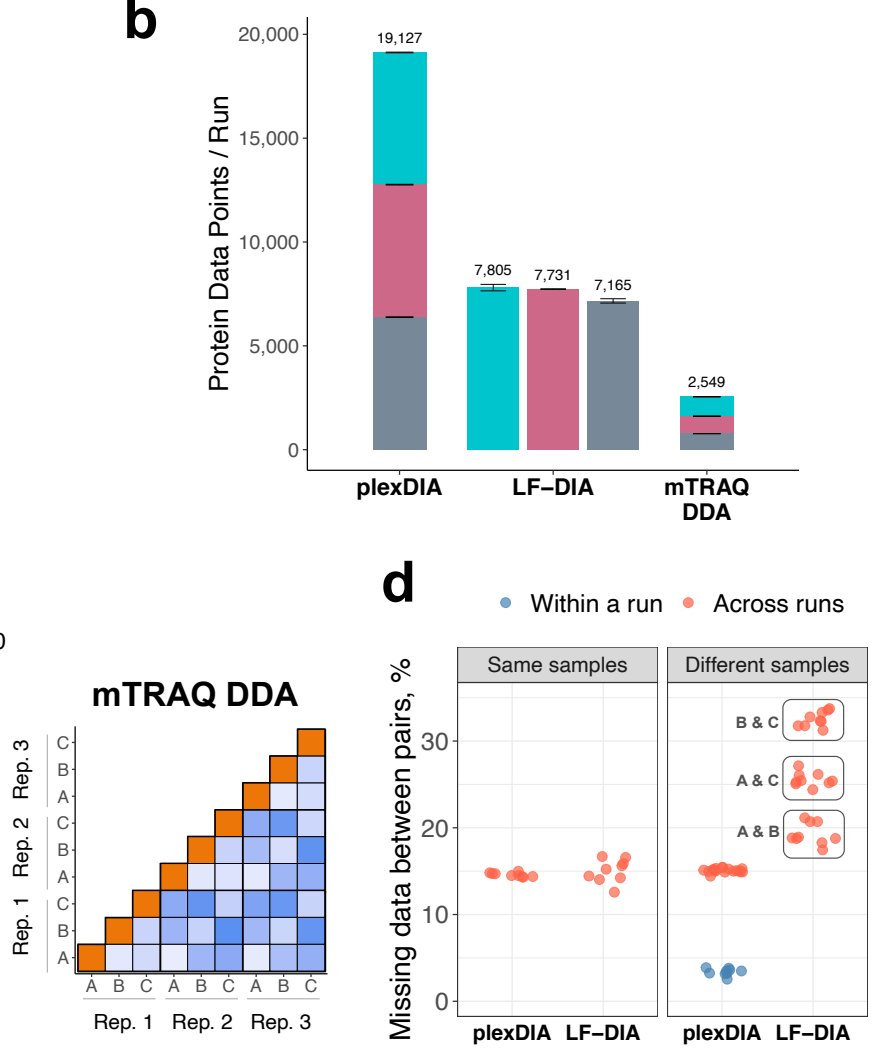

Figure 2 | plexDIA proteome coverage and overlap between samples and runs (a) Number of distinct precursors identified from 60 min active gradient runs of plexDIA, LF-DIA, and mTRAQ DDA at $1 \%$ FDR. The DIA analysis employed the V1 duty cycle shown in Fig. 1c. Each sample was analyzed in triplicate and the results displayed as mean; error bars correspond to standard deviations. (b) Total number of protein data points for plexDIA, LF-DIA, and mTRAQ DDA at $1 \%$ global protein FDR. (c) The similarity between the quantified proteins across samples is quantified by the corresponding pairwise Jaccard indices. (d) Distributions of missing data between pairs of runs of either the same sample (i.e., replicate injections) or between different samples. All analysis used match between runs. The corresponding results for the V2 duty cycle are shown in Fig. S1. 


\section{plexDIA increases proteomic overlap between samples}

Next we sought to compare the consistency of protein identification between LF-DIA and plexDIA. The systematic acquisition of ions by DIA is well established as a strategy for increasing the reproducibility of peptide identification relative to shotgun $\mathrm{DDA}^{22}$. In addition to consistent data acquisition, plexDIA may further reduce the variability between samples and runs, and thus further increase the consistency (overlap) between quantified proteins relative to LF-DIA.

Indeed, both SILAC and isobaric labeling reduce missing data by enabling the quantification of peptides identified in at least one sample from a labeled set ${ }^{16,36}$. Similarly, plexDIA takes advantage of the precisely known mass-shifts in the MS spectra to propagate peptide sequence identifications within a run. Specifically, confidently identified precursors in one channel (label) are matched to corresponding precursors in the other channels. This is the default analysis used with standards A, B and C. plexDIA has an additional mode for the special case when some proteins are present only in some samples of labeled sets. In such cases, plexDIA can enable sample specific identification for each protein by using multiple MS1- and MS2-based features to rigorously evaluate the spectral matches within a run and explicitly assign confidence for the presence of each protein in each sample. Such a special case is exampled by a plexDIA set in which one sample has both yeast and bacterial proteins while another sample has only yeast proteins, Fig. S3. These new analytical capabilities are described in the methods.

We benchmarked the consistency of identified proteins both from the repeated analysis of the same sample (such as replicate injections of sample A) and from the analysis of different samples (such as comparing samples B and C). Consistent with prior reports for DIA data completeness, both LF-DIA and plexDIA identified largely the same proteins from replicate injections, quantified by high Jaccard indices and only about $15 \%$ non-overlapping proteins, as shown in Fig. 2c,d. This overlap is comparable to the one in high-quality publicly available LF-DIA dataset by Navarro, et $a l .^{21}$ as shown in Fig. S2. The overlap between the proteins identified in distinct samples remained similarly high for plexDIA while it was significantly reduced for the LF-DIA analysis, Fig. 2c,d. This increased reproducibility for plexDIA likely arises from the fact that samples A, B, and C are analyzed in parallel as part of one set; this confers a further benefit of reduced missing data rate within a plexDIA set of only $4 \%$, Fig. $2 \mathrm{c}, \mathrm{d}$. The larger the difference in protein composition 
between two samples, the higher the fraction of missing data for LF-DIA. In contrast, the missing data for plexDIA was low across all pairs of samples, Fig. $2 \mathrm{~d}$.

\section{plexDIA increases protein ratios multiplicatively with the number of labels}

The increased consistency of protein identifications by plexDIA increases the number of proteins that can be analyzed across samples without imputation. As a result, the numbers of protein ratios quantified from the plexDIA data is comparable or exceeds the corresponding numbers for LFDIA, Fig. 3a-c. The number of protein groups quantified across all three samples (A, B and C) at $1 \%$ FDR is 5,978 for plexDIA and 5,556 for LF-DIA. Thus, a 3-plex plexDIA increased the rate of quantifying protein ratios across all 3 samples by 3.23 fold. This increased coverage with plexDIA benefits from the increased intersection between samples analyzed by plexDIA run relative to LF-DIA, Fig. 2. The improved data completeness of plexDIA is especially pronounced when comparing samples with differential protein abundances, such as samples B and C; sample C has 6-fold more E. coli and 6-fold less $S$. cerevisiae relative to sample B. As a result, LF-DIA allowed to quantify only 1,346 ratios between $E$. coli and $S$. cerevisiae proteins while plexDIA allowed to quantify 1,859 protein ratios, Fig. $3 c$.

\section{Quantitative accuracy of plexDIA is comparable to LF-DIA}

To benchmark the quantitative accuracy and precision of plexDIA and LF-DIA, we compared the measured protein ratios between pairs of samples to the ones expected from the study design, Fig. 1. Because each sample contains a known amount of E. coli, S. cerevisiae, and H. sapiens protein lysate and most peptides are unique to each species, the protein ratios between pairs of samples correspond to the corresponding mixing ratios ${ }^{21,22}$. The expected ratios allow for rigorous benchmarking of the accuracy and precision of plexDIA and LF-DIA. H. sapiens protein group ratios were excluded from analyses involving sample $\mathrm{C}$ as it would compare U-937 (A and B) to Jurkat (C) cell lines - therefore, deviations from expected ratios would be a combination of quantitative noise and cell-type specific differences in protein abundance.

For well controlled comparisons between the quantitative accuracy of LF-DIA and plexDIA, 

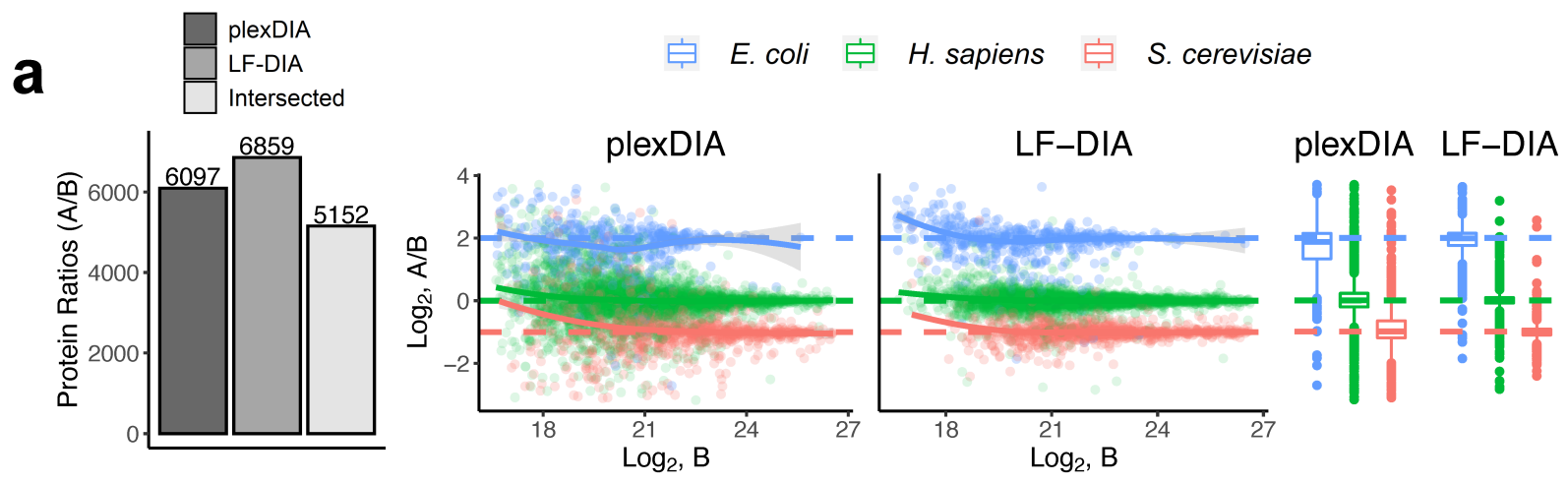

b
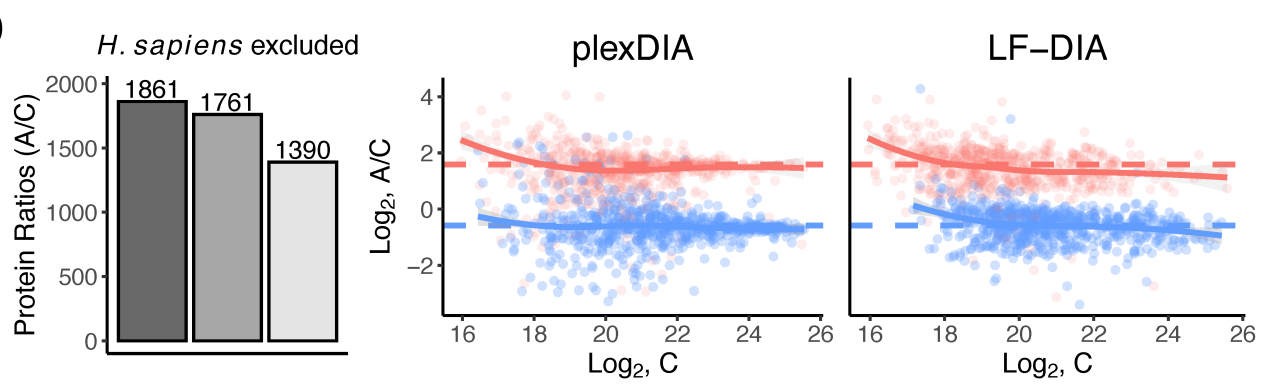

plexDIA LF-DIA

C
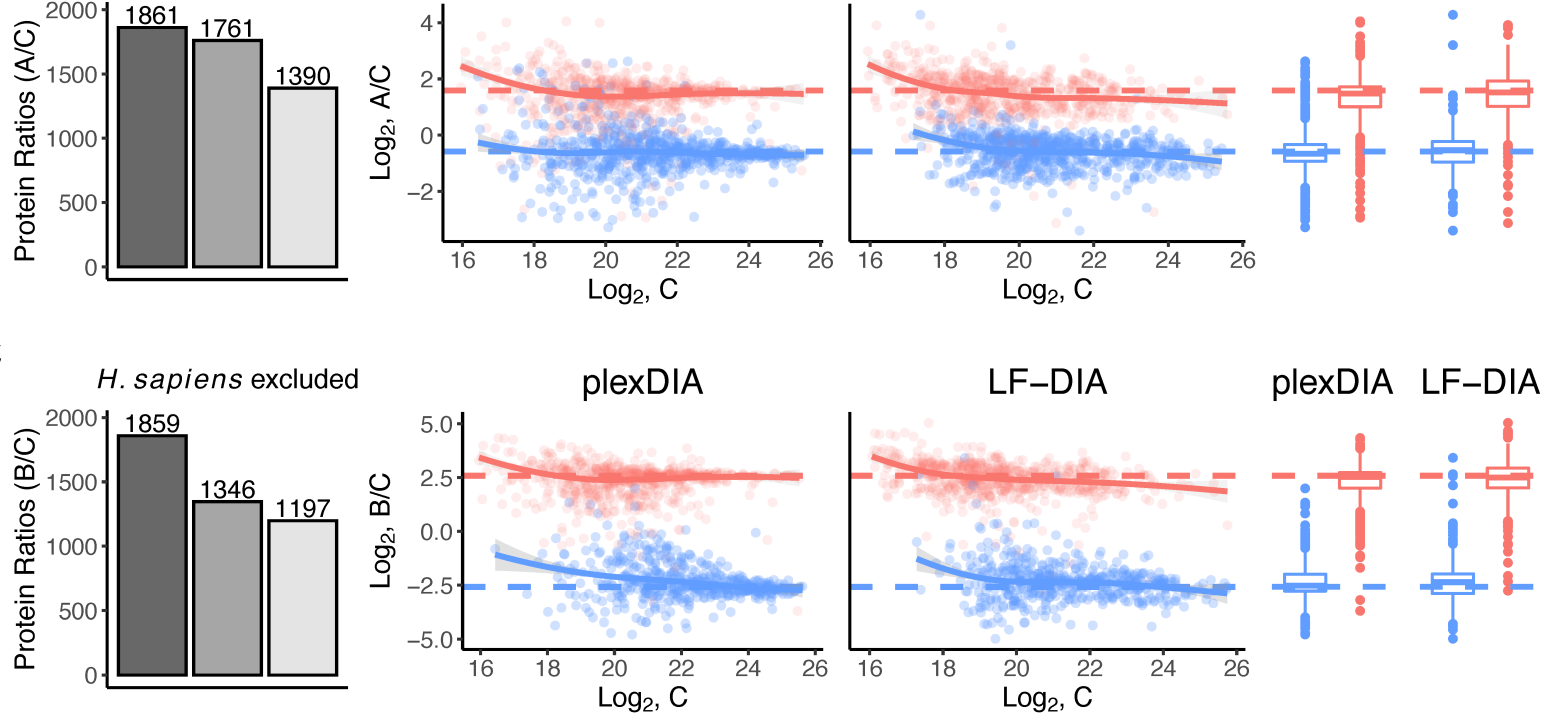

d

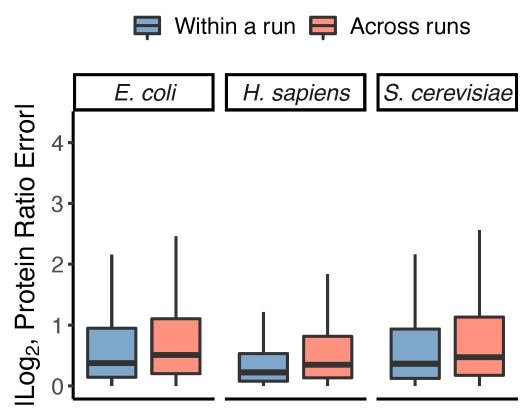

e
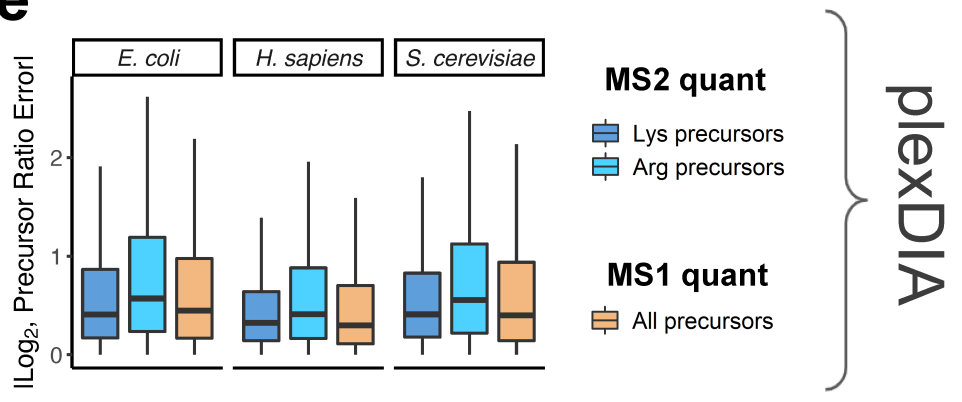

Figure 3 | Quantitative accuracy and precision of plexDIA and LF-DIA (a) Bars correspond to the number of quantified protein ratios between samples $A$ and $B$ by plexDIA, by LF-DIA, or by both methods (intersected proteins). (b) Same as (a), but for samples A and C. (c) Same as (a), but for samples B and C. The protein ratios displayed in panels a-c are estimated from a single replicate, and two more replicates are shown in Fig. S4. (d) A comparison between the accuracy of quantification within and across plexDIA sets shows similar accuracy. The absolute protein ratio errors were calculated for samples $A / B, A / C$, and $B / C$ and combined to compare ratio errors for samples within a plexDIA set (e.g., run2 A / run2 B) to samples across sets (e.g., run1 A / run2 B). The comparable accuracy within and across plexDIA is also evident in Fig. S5. (e) Absolute precursor ratio errors were calculated for samples $A / B, A / C$, and $B / C$ and combined to compare ratio errors for MS1 and MS2 quantification. The MS2 quantification of precursors having C-terminal lysine or arginine is shown separately. 
we used the set of protein ratios quantified by both methods. The comparison results from V1 are shown in Fig. 3a-c and from V2 in Fig. S6. These results indicate that on average plexDIA has comparable accuracy and precision to LF-DIA. Consistent with the expectation that labeling helps to control for nuisances, the results indicate that plexDIA quantification within a set is slightly more accurate than across sets, Fig. 3d. However, the difference is small, and accuracy across different plexDIA sets is high, Fig. S5.

By design, plexDIA allows quantifying precursors based on MS2- and MS1-level data, and we evaluated the quantitative accuracy for both levels of quantification, Fig. 3e. Since both lysine and $\mathrm{N}$-terminal amine groups are labeled by the amine-reactive mTRAQ labels, both $\mathrm{b}$ - and y-fragment ions of lysine peptides are sample-specific and thus contribute to MS2 level quantification. In contrast, only b-ions are sample-specific for arginine peptides, and thus only b-ions are used for their MS2-level quantification. As a result, the MS2-level quantification accuracy for arginine peptides is slightly lower, Fig. 3e. The small magnitude of this difference is likely attributable to the fact that mTRAQ stabilizes b-ions ${ }^{37}$. The accuracy of MS1-quantification by V1 is high for all peptides and slightly higher than the accuracy of MS2 quantification Fig. 3e. The MS2 optimized duty cycle (V2) resulted in deeper proteome coverage and lower accuracy for both LF-DIA and plexDIA, Fig. S6. However, different duty cycles implemented on different instruments will likely improve the accuracy and coverage by MS2-optimized methods.

\section{Reproducibility of plexDIA is comparable to LF-DIA}

To assess the reproducibility of plexDIA and LF-DIA quantitation, we computed the coefficient of variation $(\mathrm{CV})$ for proteins quantified in triplicate runs for each method using MaxLFQ abundance for each sample-specific protein group calculated by the DIA-NN R package ${ }^{23}$; we required each protein group to be quantified three times for plexDIA and LF-DIA, then the CVs for the overlapping protein groups $(n=13,986)$ were plotted in Fig. 4a. The results indicate that plexDIA and LF-DIA have relatively consistent quantitation and comparable quantitative reproducibility with median CVs of 0.100 and 0.108 , respectively. However, unlike accuracy, high reproducibility may be due to systematic artifacts, and thus it should be interpreted with caution. 


\section{Estimating differential protein abundance by plexDIA and LF-DIA}

We investigated the agreement of differential protein abundance between U-937 and Jurkat cell lines with plexDIA and LF-DIA. Differential protein abundance was estimated from LF-DIA data, and the differentially abundant proteins at $1 \%$ FDR were used to assess the agreement between U-937 and Jurkat protein ratios estimated by plexDIA and LF-DIA, Fig. 4b. The estimates by the two methods are similar, as indicated by a Spearman correlation of 0.81 for differentially abundant proteins $(n=1,354$ at $1 \%$ FDR), and a Spearman correlation of 0.62 for all intersected human proteins $(n=3,552)$ (Fig. 4b).

We also compared the ability of plexDIA and LF-DIA to recall true differentially abundant proteins as a function of each method's empirical FDR. Our experimental design from Fig. 1 provides strong ground truth. It dictates that between samples A and B, only S. cerevisiae and E. coli are differentially abundant because they were spiked in at different ratios (1:2 and 4:1, respectively) while human proteins are not because they are present in a 1:1 ratio and compare the same cell type (U-937 monocytes) across samples A and B. Therefore, true positives (S. cerevisiae and E. coli proteins) and true negatives (H. sapiens proteins) are known. With this prior knowledge, we compared the number of true positives for LF-DIA and plexDIA as a function of the empirical FDR, Fig. 4c. Both methods used 3 replicates and performed comparably at $1 \%$ empirical FDR, with 610 proteins and 607 proteins found to be differentially abundant for plexDIA and LF-DIA, respectively. Thus, plexDIA achieved the same statistical power as LF-DIA while using 3-times less instrument time and expense.

\section{Cell division cycle analysis with plexDIA}

Next, we applied plexDIA to quantify protein abundance across the cell division cycle (CDC) of U-937 monocyte cells. The CDC analysis allows further validation of plexDIA based on well established biological processes during the CDC while simultaneously offers the possibility of new discoveries. The ability of plexDIA to analyze small samples made it possible to isolate cells from different phases of the CDC based on their DNA content, Fig. 5a. The cell isolation used fluorescence activated cell sorting (FACS), which allowed us to analyze cell populations from G1, 

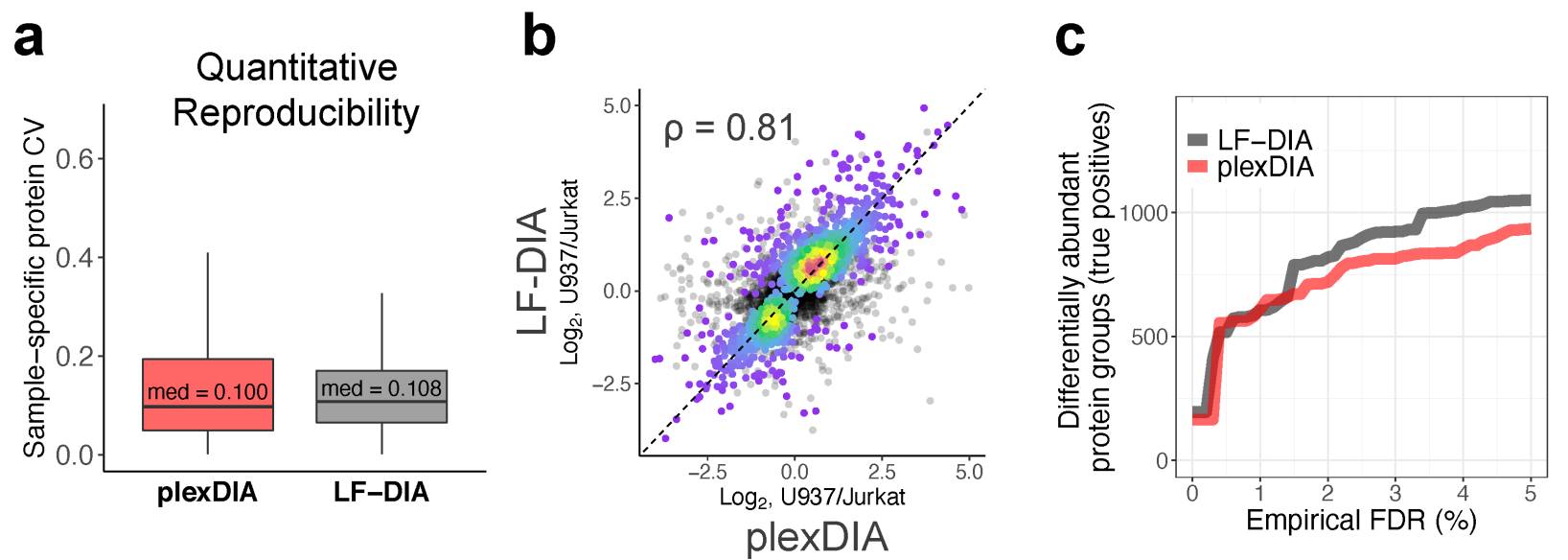

Figure 4 Using plexDIA to estimate differential protein abundance (a) Quantitative reproducibility was estimated by calculating coefficients of variation (CV) for MaxLFQ protein abundances (13,986 sample-specific protein data-points) calculated across triplicates for plexDIA and LF-DIA. (b) Proteins found to be differentially abundant between U-937 and Jurkat cells by LF-DIA were plotted as ratios of U-937/Jurkat for plexDIA and LF-DIA and colored by density. The Spearman correlation shown was calculated to quantify the agreement between the estimated relative protein levels of differentially abundant proteins at $1 \%$ FDR. Non-differentially abundant proteins are plotted in black; the Spearman correlation of all proteins $(n=3,552)$ and differentially abundant proteins at $1 \% \operatorname{FDR}(n=1,354)$ is 0.62 and 0.81 , respectively. (c) Number of differentially abundant proteins between samples $A$ and $B$ as a function of the empirical FDR. The $y$-axis shows the number of true positives (only S. cervisiae and E. coli proteins, which are differentially abundant) and the $x$-axis shows the false discovery rates estimated from the human proteins identified to be differentially abundant. The differential abundance was estimated using 3 replicates from each method, and thus LF-DIA took 3-times more instrument time per sample than plexDIA. 
$\mathrm{S}$, and G2/M phases without the artifacts associated with blocking the CDC to achieve population synchronization $^{38}$.

The peptides from the sorted cells were labeled with non-isobaric isotopologous labels, combined, and analyzed both by MS1-optimized (V1) and MS2-optimized (V2) plexDIA methods, Fig. 5a. By using different data acquisition methods, we aimed to (i) reduce systematic biases that may be shared by technical replicates and (ii) evaluate the agreement between MS1 and MS2based quantification by plexDIA in the context of a biological experiment. Analyzing the data with DIA-NN resulted in 4,344 unique protein groups quantified by both V1 and V2 samples at $1 \%$ global FDR.

To identify biological processes regulated across the phases of the CDC, we performed protein set enrichment analysis (PSEA) using data from both V1 and V2, Fig. 5b. The V1 and V2 data indicated very similar PSEA patterns and identified canonical CDC processes, such as the activation of the MCM complex during S phase, and chromatid segregation and mitotic nuclear envelope disassembly during G2/M phase, Fig. 5b. These expected CDC dynamics and the agreement between V1 and V2 results demonstrate the utility of plexDIA for biological investigations. Furthermore, the PSEA indicated metabolic dynamics in the tricarboxylic acid (TCA) cycle and fatty acid betaoxidation. These results provide direct evidence for the suggested coordination among metabolism and cell division ${ }^{39,40}$.

To further explore the proteome remodeling during the CDC, we identified differentially abundant proteins across G1, S and G2/M phase, Fig. 5c. From the 4,344 proteins quantified across $\mathrm{V} 1$ and V2, 446 proteins were found to be differentially abundant between cell cycle phases at $1 \%$ FDR. Some of these proteins are displayed in Fig. 5c organized thematically based on their functions. Consistent with results from PSEA, we find good agreement between V1 and V2 and expected changes in protein abundance, such as Cyclin A2 and ubiquitin-conjugating enzyme E2 peaking in abundance during $\mathrm{G} 2 / \mathrm{M}$ phase.

In addition to the differential abundance of classic CDC regulators, we find that some poorly characterized proteins are also differentially abundant, such as proteins CDV3 and JPT2. To further investigate these proteins, we examined the extracted ion current (XIC) for representative peptides from these proteins, Fig. 5d. The XIC demonstrate consistent quantitative trends and coelution 


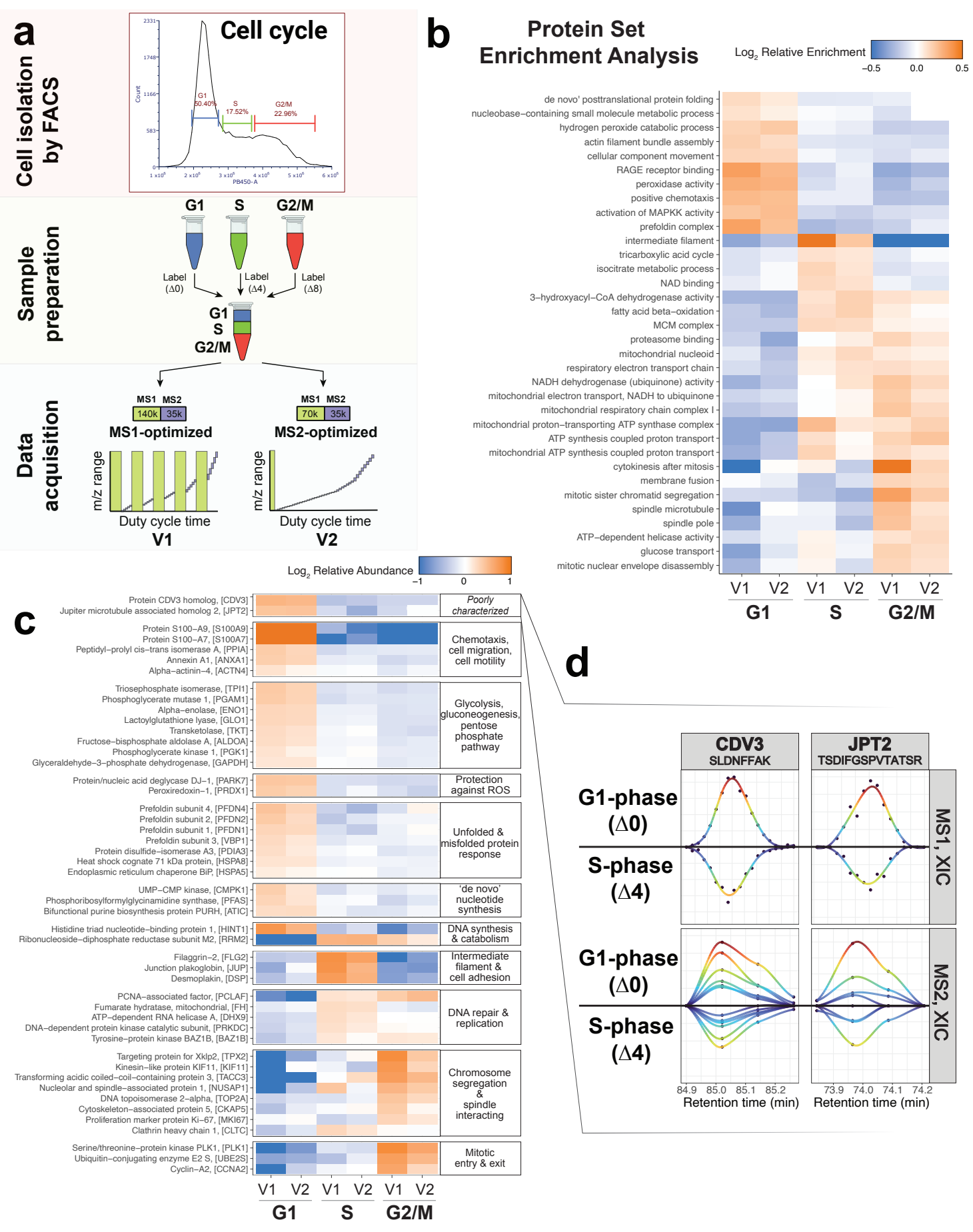

Figure 5 | Cell cycle analysis with plexDIA (a) U-937 monocytes were sorted by FACS based on DNA content to separate into $\mathrm{G} 1, \mathrm{~S}$, and G2/M cell-cycle phases; the samples were prepared as a plexDIA set, then analyzed with MS1 and MS2-optimized data acquisition methods (referred to as V1 and V2, respectively). (b) Protein set enrichment analysis of cell-cycle phases from plexDIA data. (c) A subset of proteins found to be differentially abundant at $1 \%$ FDR across cell-cycle phases were grouped by function, then plotted to show the relative abundances across cell-phases. (d) Extracted-ion chromatograms (XIC) at MS1 and MS2 for precursors from poorly characterized proteins, CDV3 and JPT2. 
among precursors and peptide fragments labeled with different mass tags. This consistency among the raw data bolsters the confidence in new few findings by plexDIA, such as differential abundance of CDV3 and JPT2.

\section{Discussion}

While multiple methods allow increasing proteomics throughput, plexDIA is distinct in simultaneously allowing high sensitivity, depth and accuracy. plexDIA enables a multiplicative increase (3-fold with 3 labels) in the rate of consistent protein quantification across limited sample amounts while preserving proteomic coverage, quantitative accuracy, precision, and reproducibility of LFDIA. Similar to other labeling methods, such as TMT-DDA, parallel analysis of multiple samples by plexDIA saves LC-MS/MS time and costs. Currently, the commercially available labels for plexDIA are low-plex (mTRAQ, TMT0/TMT/shTMT, or dimethyl labeling ${ }^{10}$ ), compared to 18 plex isobaric TMTpro labels available for $\mathrm{DDA}^{4}$. This current label disadvantage is offset by the parallel precursor analysis enabled by plexDIA. We expect that the plexDIA framework will motivate the development of higher plex labels that are optimized for different applications, such as for single-cell proteomics ${ }^{7}$.

The parallel sample and peptide analysis by plexDIA becomes increasingly important for lowly abundant samples since they require long ion accumulation times that undermine the throughput of serial acquisition methods, such as TMT-DDA ${ }^{7,41}$. Thus, plexDIA is particularly attractive for the analysis of nanogram samples as it may afford accurate and deep proteome quantification without using 2-dimensional peptides separation (offline fractionation). Indeed, plexDIA is motivated in part by ideas for achieving sensitive and multiplexed single-cell proteomics ${ }^{7,17,26}$.

Our data demonstrate that plexDIA reduces the amount of missing data between diverse samples both within and across runs. This reduction stems from buffering sample-to-sample variability in protein composition. Furthermore, we introduced an approach for matching precursors within a run, which reduce missing data to mere $4 \%$, Fig. 2. Thus, plexDIA analysis of samples with variable protein composition or abundance results in less missing data. This opens the potential for further gains. For example, small samples could be labeled then combined with a labeled carrier 
sample to improve proteomic coverage of the smaller samples. Such nonisobaric carrier design will naturally extend the isobaric carrier concept ${ }^{18,26,42}$ and its benefits to DIA analysis.

plexDIA offers a framework that may scale to $n$ labels, and thus increase throughput $n$-fold, reduce costs nearly $n$-fold, and increase the fraction of proteins quantified across all samples. Crucially, plexDIA maintains accurate quantification and good reproducibility. Here, we demonstrate this potential for $n=3$. Increasing $n$ beyond 3 offers clear benefits but also faces challenges. One challenge is the increased potential for interference, which can be resolved by increasing the resolving power of MS scans and improving data analysis. Another challenge is sampling enough ions from each peptide given the finite capacity of MS detectors, which can be relieved by sampling smaller $\mathrm{m} / \mathrm{z}$ ranges, e.g., quantification relying on small MS2 windows or split m/z ranges at MS1. The capacity of MS detectors is less limiting for small samples, such as single cells, and thus increasing the number of labels holds much potential for single-cell proteomics, as previously discussed $^{26,43}$.

The plexDIA framework intentionally uses nonisobaric isotopologous labels, which results in sample-specific precursors (allowing MS1 quantification) and in sample-specific peptide fragments (allowing MS2 quantification). This strategy is opposite to previous approaches ${ }^{31,32}$ and could in principle increase interference. Yet, this theoretical potential is effectively thwarted by our data analysis, and thus it does not significantly affect our results presented in Fig. 3. Rather, the plexDIA strategy enables quantification at the MS1 and MS2 levels, which offers advantages for at least some applications.

While here we demonstrate the utility of plexDIA to increase throughput of measuring protein abundance, it may increase the throughput of applications seeking to quantify protein interactions, conformations and activities. For example, plexDIA is readily compatible with the recently reported covalent protein painting that enables analysis of protein conformations in living cells ${ }^{44,45}$.

In this work, we have solely demonstrated the capabilities of plexDIA in providing a foldchange throughput increase for DIA proteomics, while yielding comparable data quality. While the 3-fold speed increase is already enabling for many applications, plexDIA unleashes vast opportunities offered by multiplexed proteomics combined with the latest DIA technologies, which extend far beyond just throughput and will be explored in future works currently in preparation. For 
example, plexDIA can enable significant gains in sensitivity for single-cell proteomics ${ }^{7}$. Indeed, including an isotopologous carrier channel, wherein a concentrated standard or pooled sample is used (i) to increase the sensitivity and thus identification numbers and data completeness in other channels, and (ii) to provide a reference signal for quantification. The quantitative aspect here has a double benefit. Quantification accuracy and robustness can be improved by (i) using MS1and MS2-level signals that are minimally affected by interference and by (ii) calculating quantities relative to the internal standard, which is likely to also significantly reduce the batch effects associated with LC-MS performance variation. This makes the technology introduced by plexDIA highly promising not just for very deep profiling of selected samples using offline fractionation, but also for large-scale experiments, wherein batch effects are a significant challenge. Of note, there are no fundamental limitations preventing the creation of non-isobaric labels which would allow a higher degree of multiplexing with DIA, thus enabling even higher throughput. Given these considerations, we believe that plexDIA will eventually become the predominant DIA workflow, preferable over label-free approaches for most applications.

\section{Methods}

\section{Cell culture}

U-937 (monocytes) and Jurkat (T-cells) were grown at $37^{\circ} \mathrm{C}$ in RPMI-1640 Medium (SigmaAldrich, R8758) with 10\% fetal bovine serum (Gibco, 10439016) and 1\% penicillin-streptomycin (Gibco, 15140122). Cells were harvested at a density of $10^{6}$ cells $/ \mathrm{mL}$, washed with sterile PBS, then resuspended to a concentration of $5 \times 10^{6}$ cells $/ \mathrm{mL}$ in LC-MS water and stored at $-80^{\circ} \mathrm{C}$.

E. coli and S. cerevisiae were grown at room-temperature $\left(21^{\circ} \mathrm{C}\right)$ shaking at $300 \mathrm{rpm}$ in Luria Broth (LB) and yeast-peptone-dextrose (YPD) media, respectively. Cell density was measured by OD600 and cells were harvested mid-log phase, pelleted by centrifugation, and stored at $-80^{\circ} \mathrm{C}$. 


\section{Sample Preparation}

The harvested U-937 and Jurkat cells were heated at $90^{\circ} \mathrm{C}$ in a thermal cycler for 10 min to lyse by $\mathrm{mPOP}^{46}$. Tetraethylammonium bromide (TEAB) was added to a final concentration of $100 \mathrm{mM}$ ( $\mathrm{pH} 8.5$ ) for buffering, then proteins were reduced in tris(2-carboxyethyl)phosphine (TCEP, Supelco, 646547) at $20 \mathrm{mM}$ for 30 minutes at room temperature. Iodoacetamide (Thermo Scientific, A39271) was added to a final concentration of $15 \mathrm{mM}$ and incubated at room temperature for 30 minutes in the dark. Next, Benzonase Nuclease (Millipore, E1014) was added to 0.3 units $/ \mu \mathrm{L}$, Trypsin Gold (Promega, V5280) to 1:25 ratio of substrate:protease, and LysC (Promega, VA1170) to $1: 40$ ratio of substrate:protease, then incubated at $37^{\circ} \mathrm{C}$ for 18 hours. E. coli and S. cerevisiae samples were prepared similarly; however, instead of lysis by mPOP, samples were lysed in $6 \mathrm{M}$ Urea and vortexed with acid-washed glass beads alternating between 30 seconds vortexing and 30 seconds resting on ice, repeated for a total of 5 times.

After digestion, all samples were desalted by Sep-Pak (Waters, WAT054945). Peptide abundance of the eluted digests was estimated by nanodrop A280, and then the samples were dried by speed-vacuum and resuspended in $100 \mathrm{mM}$ TEAB (pH 8.5). U-937, Jurkat, E. coli, and S. cerevisiae digests were mixed to generate three samples which we refer to as Sample A, B, and C, and the mixing ratios are described in Table S1. Samples A, B, and C were split into two groups: (i) was kept label-free, and (ii) was labeled with mTRAQ $\Delta 0, \Delta 4$, or $\Delta 8$ (SciEx, 4440015, 4427698, 4427700), respectively. An appropriate amount of each respective mTRAQ label was added to each Sample A-C, following manufacturers instructions. In short, mTRAQ was resuspended in isopropanol, then added to a concentration of $1 \mathrm{unit} / 100 \mu \mathrm{g}$ of sample and left to incubate at roomtemperature for 2 hours. We added an extra step of quenching the labeling reaction with $0.25 \%$ hydroxylamine for 1 hour at room-temperature, as is commonly done in TMT experiments where the labeling chemistry is the same ${ }^{6,41}$. After quenching, the mTRAQ-labeled samples (A-C) were pooled to produce the final multiplexed set used for benchmarking plexDIA. 


\section{Data Acquisition}

Multiplexed and label-free samples were injected at $1 \mu \mathrm{L}$ volumes via Dionex UltiMate $3000 \mathrm{UH}$ PLC to enable online $\mathrm{nLC}$ with a $25 \mathrm{~cm} \times 75 \mu \mathrm{m}$ IonOpticks Aurora Series UHPLC column (AUR2-25075C18A). These samples were subjected to electrospray ionization (ESI) and sprayed into a Thermo Q-Exactive orbitrap for MS analysis. Buffer A is made of $0.1 \%$ formic acid (Pierce, 85178 ) in LC-MS-grade water; Buffer B is made of $80 \%$ acetonitrile and $0.1 \%$ formic acid mixed with LC-MS-grade water.

The gradient used for LF-DIA is as follows: 4\% Buffer B (minutes 0-11.5), 4\%-5\% Buffer B (minutes 11.5-12), 5\%-28\% Buffer B (minutes 12-75), 28\%-95\% Buffer B (minutes 75-77), 95\% Buffer B (minutes 77-80), 95\%-4\% Buffer B (minutes 80-80.1), then hold at 4\% Buffer B until minute 95. The V1 duty cycle was comprised of 5x(1 MS1 full scan x 5 MS2 windows) as illustrated in Fig. 1b. Thus, the duty cycle has a total of 25 MS2 windows to span to full m/z scan range $(380-1370 \mathrm{~m} / \mathrm{z})$ with 0.5 Th overlap between adjacent windows. The length of the windows was variable for each subcycle (20 Th for subcycles 1-3, 40 Th for subcycle 4, and 100 Th for subcycle 5). Each MS1 full scan was conducted at 140k resolving power, 3x10 6 AGC maximum, and $500 \mathrm{~ms}$ maximum injection time. Each MS2 scan was conducted at 35k resolving power, $3 \times 10^{6}$ AGC maximum, $110 \mathrm{~ms}$ maximum injection time, and 27\% normalized collision energy (NCE) with a default charge of 2 . The RF S-lens was set to $80 \%$. The V2 duty cycle consisted of one MS1 scan conducted at 70k resolving power with a $300 \mathrm{~ms}$ maximum injection time and $3 \times 10^{6}$ AGC maximum, followed by $40 \mathrm{MS} 2$ scans at 35k resolving power with 110 ms maximum injection time and $3 \times 10^{6}$ AGC maximum. The window length for the first $25 \mathrm{MS} 2$ scans was set to 12.5 Th; the next 7 windows were $25 \mathrm{Th}$, then the last 8 windows were $62.5 \mathrm{Th}$. Adjacent windows shared a 0.5 Th overlap. All other settings were the same as the LF-DIA V1 method.

mTRAQ labeling increases hydrophobicity of peptides, which is why a higher \% Buffer B is used during the active gradient of multiplexed samples; in addition, the scan range was shifted 100 $\mathrm{m} / \mathrm{z}$ higher than LF-DIA to account for the added mass of the label. The gradient used for plexDIA is as follows: $4 \%$ Buffer B (minutes 0-11.5), 4\%-7\% Buffer B (minutes 11.5-12), 7\%-32\% Buffer B (minutes 12-75), 32\%-95\% Buffer B (minutes 75-77), 95\% Buffer B (minutes 77-80), 95\%-4\% Buffer B (minutes 80-80.1), then hold at 4\% Buffer B until minute 95. The plexDIA V1 duty cycle 
was comprised of 5x(1 MS1 full scan x 5 MS2 windows), for a total of 25 MS2 windows to span to full $\mathrm{m} / \mathrm{z}$ scan range $(480-1470 \mathrm{~m} / \mathrm{z})$ with 0.5 Th overlap between adjacent windows. The length of the windows was variable for each subcycle (20 Th for subcycles 1-3, 40 Th for subcycle 4, and $100 \mathrm{Th}$ for subcycle 5). Each MS1 full scan was conducted at 140k resolving power, $3 \times 10^{6}$ AGC maximum, and 500 ms maximum injection time. Each MS2 scan was conducted at 35k resolving power, $3 \times 10^{6}$ AGC maximum, 110 ms maximum injection time, and $27 \%$ normalized collision energy (NCE) with a default charge of 2 . The RF S-lens was set to $30 \%$. The plexDIA V2 duty cycle consisted of one MS1 scan conducted at 70k resolving power with a 300 ms maximum injection time and $3 \times 10^{6}$ AGC maximum, followed by $40 \mathrm{MS} 2$ scans at 35k resolving power with $110 \mathrm{~ms}$ maximum injection time and $3 \times 10^{6}$ AGC maximum. The window length for the first 25 MS2 scans was set to $12.5 \mathrm{Th}$; the next 7 windows were $25 \mathrm{Th}$, then the last 8 windows were 62.5 Th. Adjacent windows shared a 0.5 Th overlap. All other settings were the same as the plexDIA V1 method.

The gradient used for mTRAQ DDA is the same used for plexDIA. However, the duty cycle was a shotgun DDA method. The MS1 full scan range was 450-1600 m/z, and was performed with 70k resolving power, $3 \times 10^{6}$ AGC maximum, and $100 \mathrm{~ms}$ injection time. This shotgun DDA approach selected the top 15 precursors to send for MS2 analysis at $35 \mathrm{k}$ resolving power, $1 \times 10^{5}$ AGC maximum, $110 \mathrm{~ms}$ injection time, $0.3 \mathrm{Th}$ isolation window offset, $0.7 \mathrm{Th}$ isolation window length, $8 \times 10^{3}$ minimum AGC target, and 30 second dynamic exclusion.

\section{Spectral Library Generation}

The in silico predicted spectral library used in LF-DIA analysis was generated from Swiss-Prot $H$. sapiens, E. coli, and S. cerevisiae FASTAs by DIA-NN's deep learning-based spectra and retention time (RT), and IMs prediction. The spectral library used for plexDIA benchmarking was created in a similar process, with the exception of a few additional commands entered into the DIA-NN command line GUI: 1) \{-fixed-mod mTRAQ 140.0949630177, nK\}, 2) \{-original-mods\}, and 3) $\{-\min -\mathrm{fr} 16\}$ 


\section{plexDIA module in DIA-NN}

A distinct feature of DIA-MS proteomics is the complexity of produced spectra, which are a mixture of fragments ions originating from multiple co-isolated precursors. This complexity has necessitated the rise of a variety of highly sophisticated algorithms for DIA data processing. Current DIA software, such as DIA-NN ${ }^{23}$, aims to find peak groups in the data that best match the theoretical information about such peptide properties as the MS/MS spectrum, the retention time and the ion mobility. Once identified correctly, the peak group, that is the set of extracted ion chromatograms of the precursor and its fragments in the vicinity of the elution apex, allows to integrate either the MS1- or MS2-level signals to quantify the precursor, which is the ultimate purpose of the workflow.

Similar to match-between-runs (MBR) algorithms, plexDIA data provide the opportunity to match corresponding ions, in this case between the same peptide labeled with different mass tags. However, the use of isotopologous mass tags, such as mTRAQ, allows to match the retention times within a run with much higher accuracy than what can be achieved across runs. Thus, the sequence propagation can be more sensitive and reliable than with $\mathrm{MBR}^{7}$. This allows to enhance sequence identifications analogously to the isobaric carrier concept introduced by TMTbased single-cell workflows ${ }^{42,47}$. With the isobaric carrier approach, a carrier channel is loaded with a relatively high amount of peptides originating from a pooled sample that facilities peptide sequence identification ${ }^{18,26}$. We implemented a similar approach in the plexDIA module integrated in DIA-NN. Once a peptide is identified in one of the channels, this allows to determine its exact retention time apex, which in turn helps identify and quantify the peptide in all of the channels by integrating the respective precursor (MS1) or fragment ion (MS2) signals.

Apart from the identification performance, plexDIA also can increase quantification accuracy. The rich complex data produced by DIA promotes more accurate quantification because of algorithms that select signals from MS/MS fragment ions which are affected by interferences to the least extent ${ }^{23}$. For LF-DIA, DIA-NN selects fragments in a cross-run manner: fragments which tend to correlate well with other fragments across runs are retained, while those which often exhibit poor correlations due to interferences are excluded from quantification. While this approach yields good results, a limitation remains for LF-DIA: fragment ions only affected by interferences 
in a modest proportion of runs are still used for quantification, thus undermining the reliability of the resulting quantities in those runs. Here plexDIA provides a unique advantage. Theoretically, a single MS1- or MS2-level signal with minimal interference is sufficient to calculate the quantitative ratio between the channels. In this case, if low interference quantification is possible in at least one 'best' channel, this quantity can be multiplied by the respective ratios across other channels to obtain accurate estimates of quantities in all channels that share at least one low interference signal with this 'best' channel. This idea is implemented in DIA-NN to produce 'translated' quantities, which have been corrected by using ratios of high quality MS1 or MS2 signals between channels.

\section{Data Analysis with DIA-NN}

DIA-NN (version 1.8.1 beta 7) was used to search LF-DIA and plexDIA raw files. All LF-DIA benchmarking raw files were searched together with match between runs (MBR) if the same duty cycle was used; likewise, all plexDIA benchmarking raw files were searched together with MBR if the same duty cycle was used.

DIA-NN search settings: Library Generation was set to “IDs, RT, \& IM Profiling”, Quantification Strategy was set to "Peak height", scan window $=1$, Mass accuracy $=10 \mathrm{ppm}$, and MS1 accuracy = 5 ppm, "Remove likely interferences", "Use isotopologues", and "MBR" were enabled. Additional commands entered into the DIA-NN command line GUI for plexDIA: 1) \{-fixed-mod mTRAQ 140.0949630177, nK\}, 2) \{-channels mTRAQ, 0:0.0, 4:4.0070994, 8:8.0141988132\}, 3) $\{$-original-mods $\}$, 4) $\{$-peak-translation $\}$, 5) $\{$-ms 1-isotope-quant $\}$, and 6) $\{$ ms 1-subtract 2$\}$. For LF-DIA, only the following additional commands were used: 1) \{-original-mods $\}$ and 2) $\{$-peak-translation $\}$.

MaxLFQ abundance for protein groups was calculated based on MS1 intensities (specifically the "MS1 Area" column output by DIA-NN) using the DIA-NN R package ${ }^{23}$ for data acquired with the V1 method. However, for data acquired using the V2 method, MS2 quantitation (specifically the "Precursor Translated" column output by DIA-NN) was used for quantitation. These protein abundances were used to calculate protein ratios across samples, which were normalized by subsetting human proteins (which are present in a 1:1 ratio, theoretically) and multiplying by a scalar such that the human protein ratios were centered on 1, and thus the other species (E. coli, S. 
cerevisiae) would be systematically shifted to account for any small loading differences across samples.

\section{Differential protein abundance testing}

Differential protein abundance testing was performed using precursor-level quantitation. To account for variation in sample loading amounts, precursors from each sample were normalized to their sample-median. Then, each precursor was normalized by its mean across samples to convert it to relative levels. The normalized relative precursor intensities from different replicates were grouped by their corresponding protein groups and compared by a t-test (Fig. 4b,c) or ANOVA (Fig. 5c) to estimate the significance of differential protein abundance across samples/conditions. This comparison captures both the variability between different replicates and different peptides originating from the same protein. To correct for multiple hypotheses testing, we used the Benjamini-Hochberg $(\mathrm{BH})$ method to estimate q-values for differential abundance of proteins and protein sets.

\section{Cell division cycle, FACS and sample preparation}

U-937 monocytes were grown as described above, harvested and aliquoted to a final $1 \mathrm{~mL}$ suspension of approximately $1 \times 10^{6}$ cells in RPMI-1640 Medium. Then DNA was stained by incubating the cells with Vybrant DyeCycle Violet Stain (Invitrogen, V35003) at a final concentration of 5 $\mu \mathrm{M}$ in the dark for 30 minutes at $37^{\circ} \mathrm{C}$, as per the manufacturer's instructions. Next, the cells were centrifuged, then resuspended in PBS to a density of $1 \times 10^{6}$ cells $/ \mathrm{mL}$. The cell suspension was stored on ice and protected from light until sorting began.

The cells were then sorted with a Beckman CytoFLEX SRT. The population of U-937s was gated to select singlets based on FSC-A and FSC-H, this population of singlets was then subgated based on DNA content using the PB-450 laser $(\mathrm{ex}=405 \mathrm{~nm} / \mathrm{em}=450 \mathrm{~nm})$. The G1 population is the most abundant population in actively dividing cells, and the G2/M populations should theoretically have double the intensity (DNA content), while the S-phase lies in between. Populations of G1, S, and G2 cells were collected based on these subgates and sorted into $2 \mathrm{~mL}$ 
Eppendorf tubes.

Post-sorting, the cells were centrifuged at $300 \mathrm{~g}$ for 10 minutes, PBS was removed, then the cells were resuspended in $20 \mu \mathrm{L}$ HPLC water to reach a density of approximately 4,000 cells/ $\mu \mathrm{L}$. The cell suspensions were lysed using the Minimal ProteOmic sample Preparation (mPOP) method, which involves freezing at $-80^{\circ} \mathrm{C}$ and then heating to $90^{\circ} \mathrm{C}$ for 10 minutes ${ }^{46}$. Next, the cell lysates were prepared exactly as described in the "Sample Preparation" section. In brief, the cell lysate was buffered with $100 \mathrm{mM}$ TEAB (pH 8.5), then proteins were reduced with $20 \mathrm{mM}$ TCEP for 30 minutes at room temperature. Next, iodoacetamide was added to a final concentration of $15 \mathrm{mM}$ and incubated at room temperature for 30 minutes in the dark, then Benzonase Nuclease was added to 0.3 units $/ \mu \mathrm{L}$. Trypsin Gold and LysC to were then added to the cell lysate at 1:25 and 1:40 ratio of protease:substrate, then the samples were incubated at $37^{\circ} \mathrm{C}$ for 18 hours. After digestion, the peptides were desalted by stage-tipping with C18 extraction disks (Empore, 66883-U) to remove any remaining salt that was introduced during sorting ${ }^{48}$. G1 cells were labeled with mTRAQ $\Delta 0$, S cells were labeled with mTRAQ $\Delta 4$, and G2/M cells were labeled with $\Delta 8$, then combined to form a plexDIA set. The combined set was analyzed with 2 hour active gradients of MS1 (V1) and MS2-optimized (V2) methods as described in the "Data acquisition" section.

\section{Statistical analysis}

The quantitative comparisons between LF-DIA and plexDIA throughout this article are for intersected sets of proteins so that the results would not be influenced by proteins analyzed only by one method and not the other. For examples, compared distributions were for the same set of proteins to avoid "survival biases" 49 .

\section{Data Availability}

The raw data and search results are available at MassIVE: MSV000088302 Data, code \& protocols are also available at plexDIA.slavovlab.net

Acknowledgments: We thank A. Petelski, for discussions and constructive comments. This work was funded by a New Innovator Award from the NIGMS from the National Institutes of Health 
to N.S. under Award Number DP2GM123497, an Allen Distinguished Investigator award through The Paul G. Allen Frontiers Group to N.S., a Seed Networks Award from CZI CZF2019-002424 to N.S. This work received further support from the Francis Crick Institute, which receives its core funding from Cancer Research UK (FC001134), the UK Medical Research Council (FC001134), and the Wellcome Trust (FC001134 and IA 200829/Z/16/Z), as well as the European Research Council (SyG 951475 to M.R.). The work was further supported by the German Federal Ministry of Education and Research (BMBF), as part of the National Research Node 'Mass spectrometry in Systems Medicine' (MSCoresys), under grant agreements 031L0220A to M.R. and 161L0221 to V.D..

Competing Interests: The authors declare that they have no competing financial interests.

Correspondence: Correspondence and materials requests should be addressed to nslavov@alum.mit.edu

\section{Author Contributions}

Experimental design: J.D., N.S. and V.D.

LC-MS/MS: J.D., G.H. and H.S.

Sample preparation: J.D. and A.L.

Raising funding: N.S., M.R. and V.D.

Supervision: N.S. and V.D.

Data analysis: J.D., V.D., and N.S.

Initial draft: J.D., V.D., and N.S.

Writing: All authors contributed to writing the paper and approved the final manuscript. 


\section{References}

1. Bekker-Jensen, D. B. et al. An optimized shotgun strategy for the rapid generation of comprehensive human proteomes. Cell systems 4, 587-599 (2017).

2. Friedrich, C. et al. Comprehensive micro-scaled proteome and phosphoproteome characterization of archived retrospective cancer repositories. Nature communications 12, 1-15 (2021).

3. Xuan, Y. et al. Standardization and harmonization of distributed multi-center proteotype analysis supporting precision medicine studies. Nature Communications 11, 5248 (2020).

4. Li, J. et al. TMTpro-18plex: The Expanded and Complete Set of TMTpro Reagents for Sample Multiplexing. en. J. Proteome Res. 20, 2964-2972 (May 2021).

5. Messner, C. B. et al. Ultra-fast proteomics with Scanning SWATH. Nature Biotechnology. https://doi.org/10.1038/s41587-021-00860-4 (2021).

6. Petelski, A. A. et al. Multiplexed single-cell proteomics using SCoPE2. Nature Protocols, doi: 10.1038/s41596-021-00616-z. https : / / doi .org/10 . 1038 / s41596-021$00616-z(2021)$.

7. Slavov, N. Driving Single Cell Proteomics Forward with Innovation. Journal of Proteome Research, doi: 10.1021/acs.jproteome.1c00639m. https:// doi .org/10.1021/acs . jproteome.1c00639m(2021).

8. Slavov, N. Increasing proteomics throughput. Nature Biotechnology 39, 809-810. https : //doi.org/10.1038/s41587-021-00881-z (2021).

9. Slavov, N. Unpicking the proteome in single cells. Science 367, 512-513 (2020).

10. Boersema, P. J., Raijmakers, R., Lemeer, S., Mohammed, S. \& Heck, A. J. Multiplex peptide stable isotope dimethyl labeling for quantitative proteomics. Nature protocols 4, 484-494 (2009).

11. Zhang, Y., Fonslow, B. R., Shan, B., Baek, M.-C. \& Yates III, J. R. Protein analysis by shotgun/bottom-up proteomics. Chemical reviews 113, 2343-2394 (2013).

12. Petelski, A. A. \& Slavov, N. Analyzing ribosome remodeling in health and disease. Proteomics 20, 2000039 (2020).

13. Mertins, P. et al. iTRAQ labeling is superior to mTRAQ for quantitative global proteomics and phosphoproteomics. Molecular \& Cellular Proteomics 11 (2012).

14. O’Connell, J. D., Paulo, J. A., O’Brien, J. J. \& Gygi, S. P. Proteome-Wide Evaluation of Two Common Protein Quantification Methods. Journal of Proteome Research 17. PMID: 29635916, 1934-1942 (2018).

15. Muntel, J. et al. Comparison of Protein Quantification in a Complex Background by DIA and TMT Workflows with Fixed Instrument Time. Journal of Proteome Research 18. PMID: 30726097, 1340-1351 (2019).

16. Rauniyar, N. \& Yates III, J. R. Isobaric labeling-based relative quantification in shotgun proteomics. Journal of proteome research 13, 5293-5309 (2014). 
17. Specht, H. \& Slavov, N. Transformative opportunities for single-cell proteomics. Journal of Proteome Research 17, 2563-2916 (8 June 2018).

18. Specht, H. \& Slavov, N. Optimizing Accuracy and Depth of Protein Quantification in Experiments Using Isobaric Carriers. Journal of Proteome Research 20. PMID: 33190502, 880887 (2021).

19. Venable, J. D., Dong, M.-Q., Wohlschlegel, J., Dillin, A. \& Yates, J. R. Automated approach for quantitative analysis of complex peptide mixtures from tandem mass spectra. en. Nature Methods 1, 39-45. ISSN: 1548-7105. (2020) (Oct. 2004).

20. Gillet, L. C. et al. Targeted data extraction of the MS/MS spectra generated by data-independent acquisition: a new concept for consistent and accurate proteome analysis. Molecular \& Cellular Proteomics 11 (2012).

21. Navarro, P. et al. A multicenter study benchmarks software tools for label-free proteome quantification. eng. Nature Biotechnology 34, 1130-1136. ISSN: 1546-1696 (Nov. 2016).

22. Fernández-Costa, C. et al. Impact of the identification strategy on the reproducibility of DDA and DIA results. Journal of proteome research 19, 3153-3161. (2021) (2020).

23. Demichev, V., Messner, C. B., Vernardis, S. I., Lilley, K. S. \& Ralser, M. DIA-NN: neural networks and interference correction enable deep proteome coverage in high throughput. Nature methods 17, 41-44 (2020).

24. Sinitcyn, P. et al. MaxDIA enables library-based and library-free data-independent acquisition proteomics. Nature Biotechnology, 1-11 (2021).

25. Demichev, V. et al. High sensitivity dia-PASEF proteomics with DIA-NN and FragPipe. bioRxiv (2021).

26. Slavov, N. Single-cell protein analysis by mass spectrometry. Current Opinion in Chemical Biology 60, 1-9. ISSN: 1367-5931 (2020).

27. Minogue, C. E. et al. Multiplexed Quantification for Data-Independent Acquisition. Analytical Chemistry 87, 2570-2575 (2015).

28. Liu, Y. et al. Systematic proteome and proteostasis profiling in human Trisomy 21 fibroblast cells. Nature communications 8, 1-15 (2017).

29. Pino, L. K., Baeza, J., Lauman, R., Schilling, B. \& Garcia, B. A. Improved SILAC Quantification with Data-Independent Acquisition to Investigate Bortezomib-Induced Protein Degradation. Journal of Proteome Research $\mathbf{0}$, null (0).

30. Zhong, X. et al. Mass Defect-Based DiLeu Tagging for Multiplexed Data-Independent Acquisition. en. Anal. Chem. 92, 11119-11126 (Aug. 2020).

31. Tian, X., de Vries, M. P., Permentier, H. P. \& Bischoff, R. A Versatile Isobaric Tag Enables Proteome Quantification in Data-Dependent and Data-Independent Acquisition Modes 2020.

32. Tian, X., de Vries, M. P., Permentier, H. P. \& Bischoff, R. The Isotopic Ac-IP Tag Enables Multiplexed Proteome Quantification in Data-Independent Acquisition Mode. en. Anal. Chem. (May 2021). 
33. Salovska, B. et al. Isoform-resolved correlation analysis between mRNA abundance regulation and protein level degradation. Molecular systems biology 16, e9170 (2020).

34. Haynes, S. E., Majmudar, J. D. \& Martin, B. R. DIA-SIFT: A Precursor and Product Ion Filter for Accurate Stable Isotope Data-Independent Acquisition Proteomics. Analytical Chemistry 90, 8722-8726 (2018).

35. Salovska, B., Li, W., Di, Y. \& Liu, Y. BoxCarmax: a high-selectivity data-independent acquisition mass spectrometry method for the analysis of protein turnover and complex samples. bioRxiv (2020).

36. Cox, J. \& Mann, M. MaxQuant enables high peptide identification rates, individualized ppbrange mass accuracies and proteome-wide protein quantification. Nature biotechnology 26, 1367-1372 (2008).

37. Kang, U.-B., Yeom, J., Kim, H. \& Lee, C. Quantitative Analysis of mTRAQ-Labeled Proteome Using Full MS Scans. Journal of Proteome Research 9, 3750-3758. https : / / doi . org/10.1021/pr9011014(2010).

38. Cooper, S. The synchronization manifesto: a critique of whole-culture synchronization. The FEBS Journal 286, 4650-4656 (2019).

39. Aguilar, V. \& Fajas, L. Cycling through metabolism. EMBO molecular medicine 2, 338-348 (2010).

40. Slavov, N. \& Botstein, D. Coupling among growth rate response, metabolic cycle, and cell division cycle in yeast. Molecular Biology of the Cell 22, 1997-2009 (2011).

41. Specht, H. et al. Single-cell proteomic and transcriptomic analysis of macrophage heterogeneity using SCoPE2. Genome Biology 22 (2021).

42. Budnik, B., Levy, E., Harmange, G. \& Slavov, N. SCoPE-MS: mass-spectrometry of single mammalian cells quantifies proteome heterogeneity during cell differentiation. Genome Biology 19, 161 (2018).

43. Aebersold, R. \& Mann, M. Mass-spectrometric exploration of proteome structure and function. en. Nature 537, 347-355. ISSN: 1476-4687. https : / / www . nature. com/articles / nature1 9949 (2018) (Sept. 2016).

44. Bamberger, C. et al. Protein Footprinting via Covalent Protein Painting Reveals Structural Changes of the Proteome in Alzheimer's Disease. en. J. Proteome Res. (Apr. 2021).

45. Slavov, N. Measuring Protein Shapes in Living Cells. Journal of Proteome Research 20. PMID: 33988997, 3017-3017 (2021).

46. Specht, H. et al. Automated sample preparation for high-throughput single-cell proteomics. bioRxiv 10.1101/399774. https: / / doi.org/10.1101/399774 (2018).

47. Budnik, B., Levy, E., Harmange, G. \& Slavov, N. Mass-spectrometry of single mammalian cells quantifies proteome heterogeneity during cell differentiation. bioRxiv 1, DOI: 10.1101/102681 (2017). 
48. Keshishian, H. et al. Quantitative, multiplexed workflow for deep analysis of human blood plasma and biomarker discovery by mass spectrometry. en. Nature Protocols 12, 1683-1701. (2021) (Aug. 2017).

49. Huffman, G., Chen, A. T., Specht, H. \& Slavov, N. DO-MS: Data-Driven Optimization of Mass Spectrometry Methods. J. of Proteome Res. 18, 2493-2500 (6 2019). 


\section{Supplementary Figures}

\section{Increasing the throughput of sensitive proteomics by plexDIA}

Jason Derks, ${ }^{1,2,3}$ Andrew Leduc, ${ }^{1,2,3}$ R. Gray Huffman, ${ }^{1,2,3}$ Harrison Specht, ${ }^{1,2,3}$ Markus Ralser, ${ }^{4,5}$ Vadim Demichev ${ }^{4, \bowtie}$ \& Nikolai Slavov ${ }^{1,2,3, \varpi}$

${ }^{1}$ Department of Bioengineering, Northeastern University, Boston, MA 02115, USA

${ }^{2}$ Barnett Institute, Northeastern University, Boston, MA 02115, USA

${ }^{3}$ Department of Chemistry and Chemical Biology, Northeastern University, Boston, MA 02115, USA

${ }^{4}$ Charité - Universitätsmedizin Berlin, Germany

${ }^{5}$ Molecular Biology of Metabolism Laboratory, The Francis Crick Institute, London, UK

$\bowtie$ Correspondence: nslavov@northeastern.edu and vadim.demichev@charite.de

$\in$ Data, code \& protocols: plexDIA.slavovlab.net 


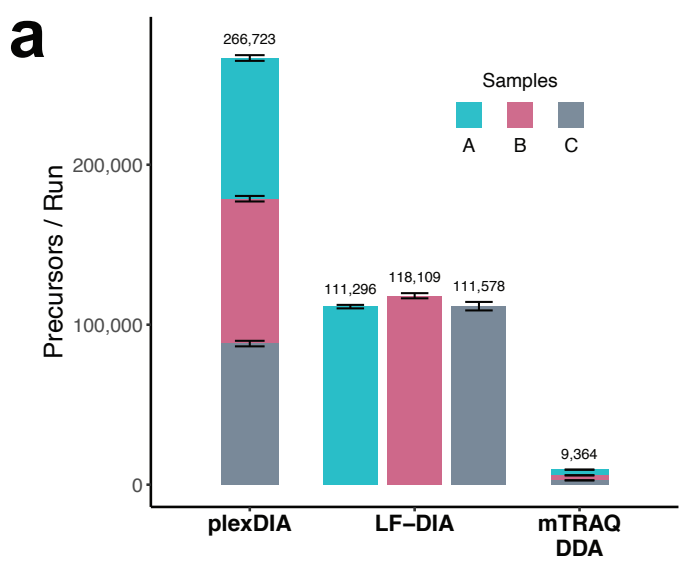

C

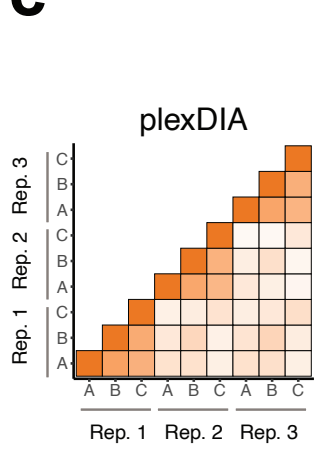

Protein Jaccard Index $\begin{array}{lll}0.6 & 0.8 & 1.0\end{array}$

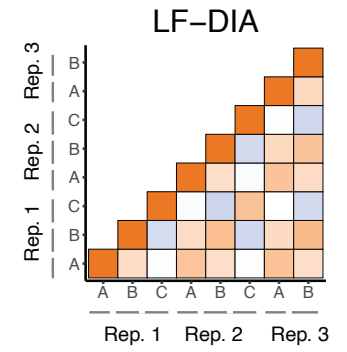

b

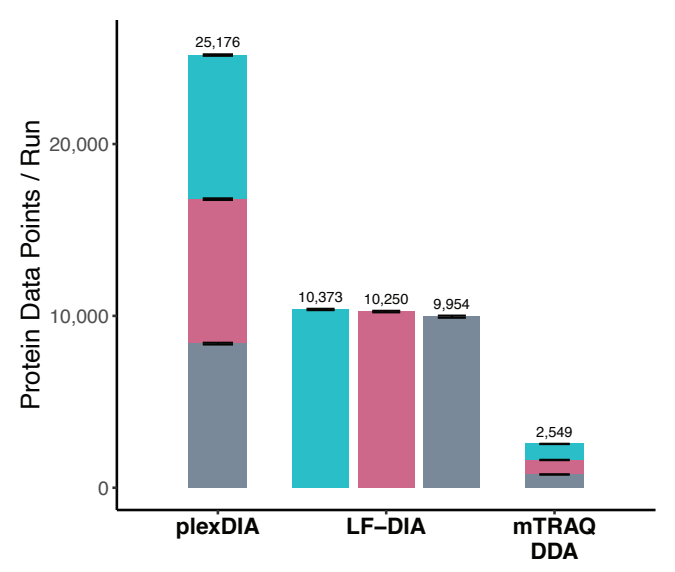

d

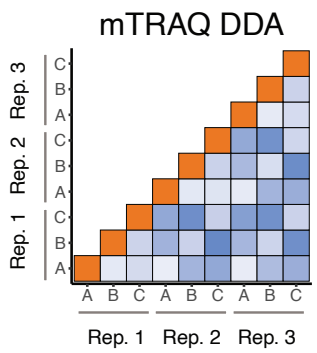

- Within a run - Across runs

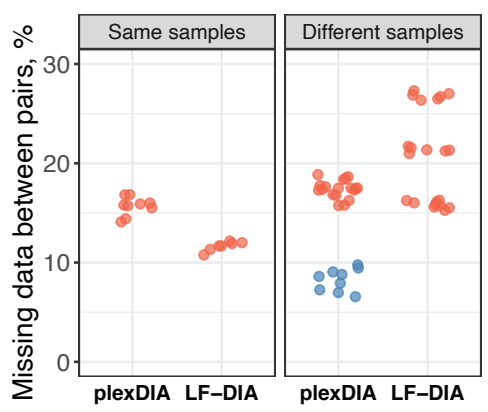

Figure S1 | plexDIA proteomic coverage and data completeness for V2 (a) Number of distinct precursors identified from 60min active gradient runs for plexDIA, LF-DIA, and shotgun-DDA of mTRAQ at $1 \%$ FDR. The DIA analysis used the V2 method, an MS2-optimized data acquisition cycle shown in Fig. 1. Each sample was analyzed in triplicate and the results displayed as mean; error bars correspond to standard deviations. (b) Total number of protein data points for plexDIA, LF-DIA, and mTRAQ DDA at $1 \%$ global protein FDR. (c) We compute pairwise Jaccard indices to compare pairwise data completeness between plexDIA, LF-DIA and shotgun DDA for mTRAQ. All DIA data were analyzed using match between runs. (d) Distributions of missing data between pairs of runs of either the same sample (i.e., replicate injections) or between different samples 

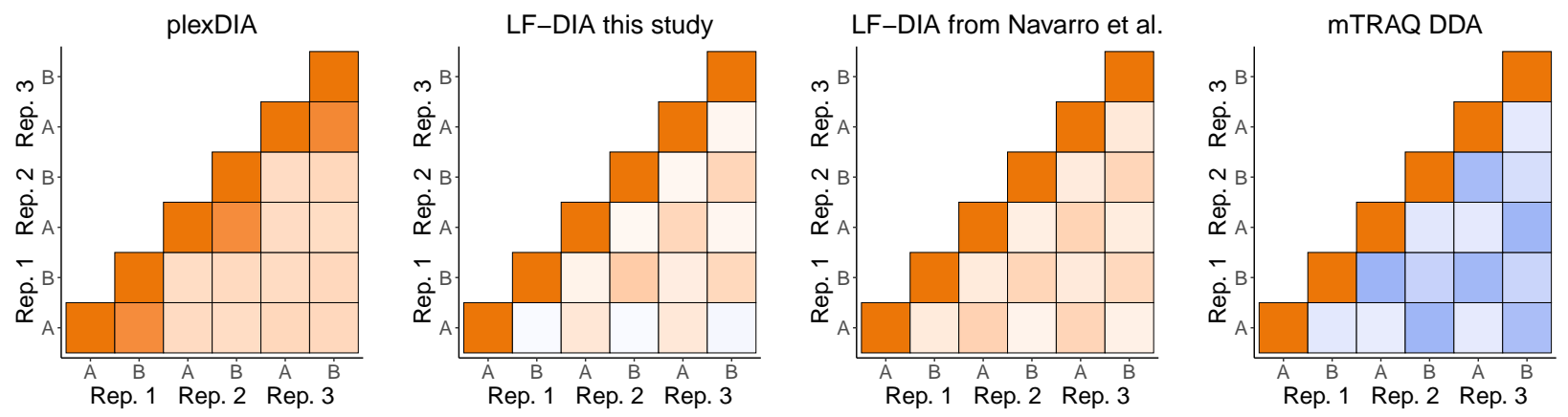

Figure S2 $\mid$ Comparison of proteomic overlap between our runs to a high quality DIA dataset (Navarro et al. ${ }^{21}$ ) DIA runs (including raw data from Navarro et al.) were searched with DIA-NN using match between runs. Results indicate that the data completeness is from LF-DIA in this study is comparable to other high quality LF-DIA datasets. 
a

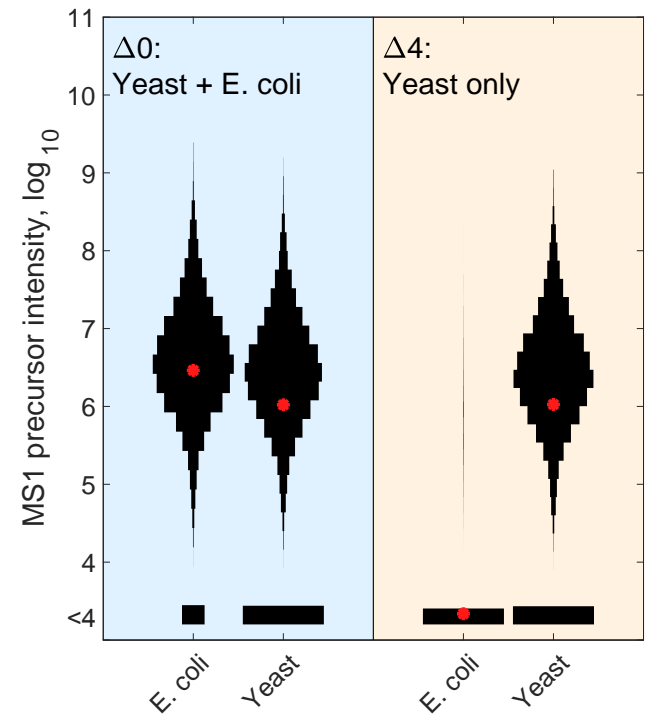

b

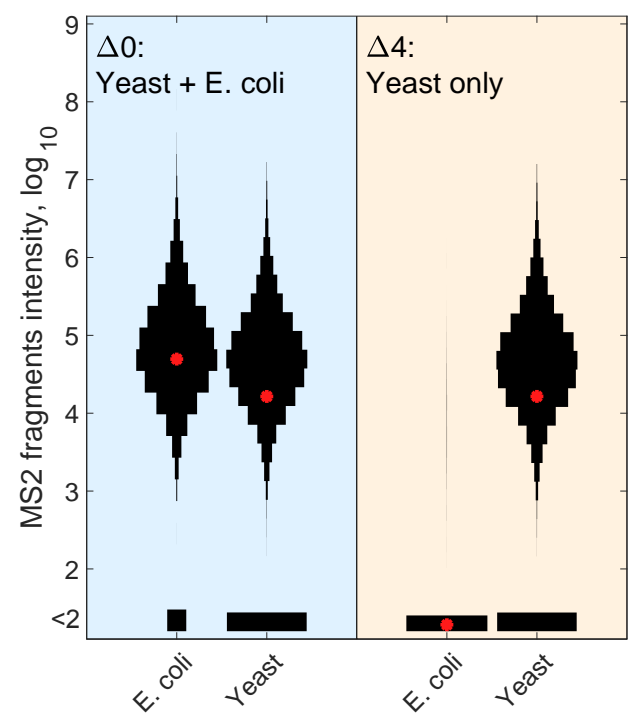

Figure S3 | plexDIA analysis proteins present only in one samples but missing from another. We sought to test identification propagation by plexDIA for the case when proteins are present only in some samples and not in others. To do so, we prepared a standard in which one sample (labeled with mTRAQ $\Delta 0$ ) had both $0.5 \mu \mathrm{g} \mathrm{E}$. coli and $0.5 \mu \mathrm{g} \mathrm{S}$. cerevisiae while another (labeled with mTRAQ $\Delta 4$ ) had only $0.5 \mu \mathrm{g} \mathrm{S}$. cerevisiae. The combined set was analyzed by plexDIA using the V1 method. (a) Distributions of raw MS1 precursor intensity for E. coli and S. cerevisiae precursors at translated q-value $<0.01$. (b) Distributions of raw MS2 quantification of precursors filtered for translated q-value $<0.01$. The red asterisks correspond to the means of the distributions. 


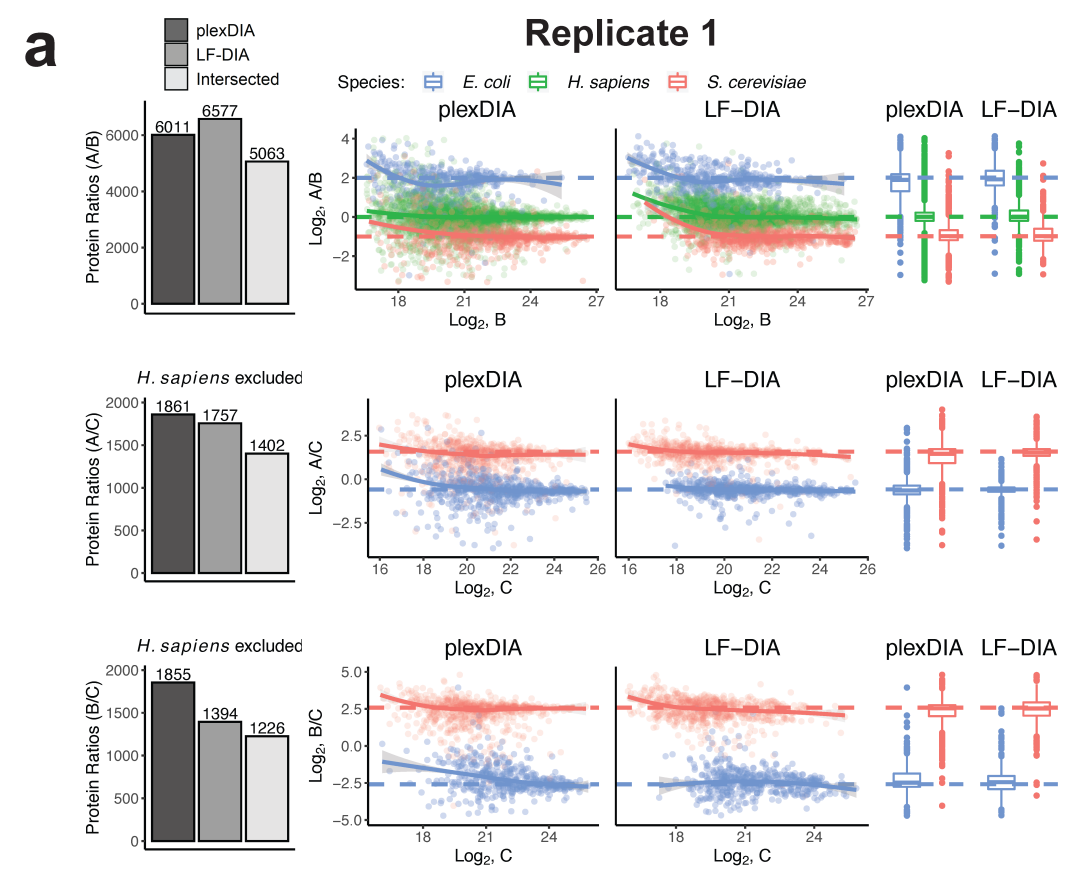

b

Replicate 3
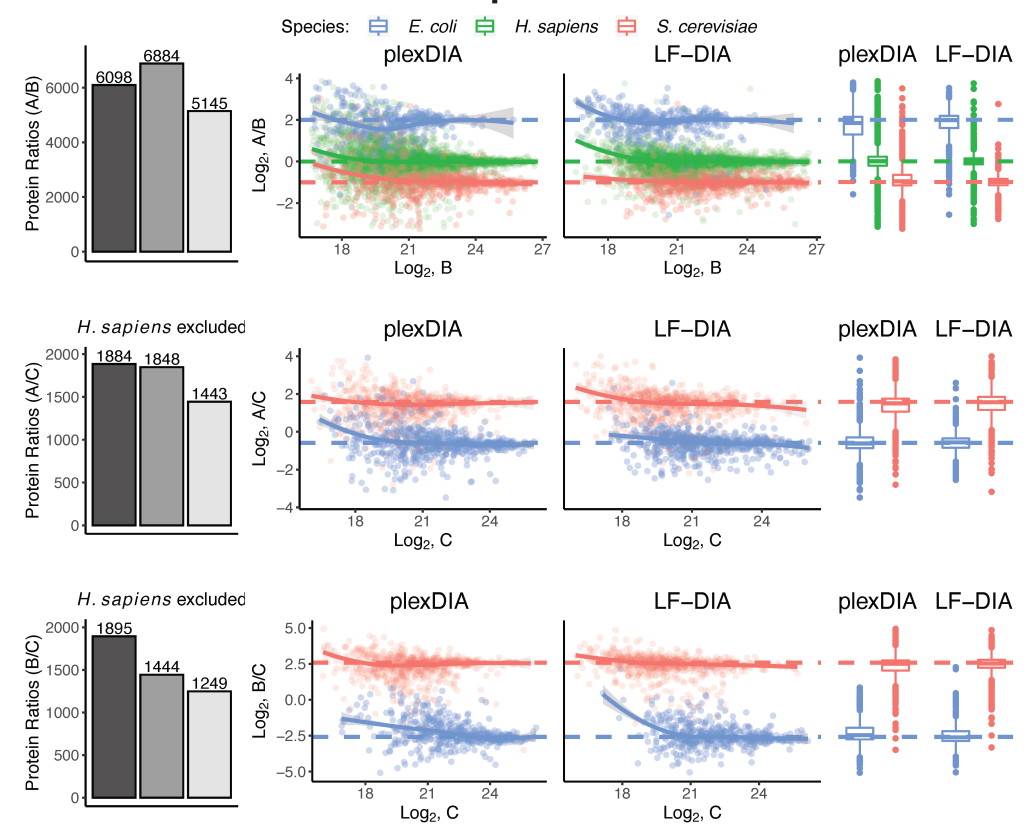

Figure S4 | Quantitative accuracy for DIA replicates using V1 Similar to main Figure 3, we display the results from the other replicates. (a) Figures are the same as shown in Figure 3 of the main text, with the exception that this shows the first replicate of plexDIA and the first replicate of samples A, B, C for LF-DIA. (b) Same as (a), but for the third replicate of plexDIA and LF-DIA samples A, B, C. 


\section{a}
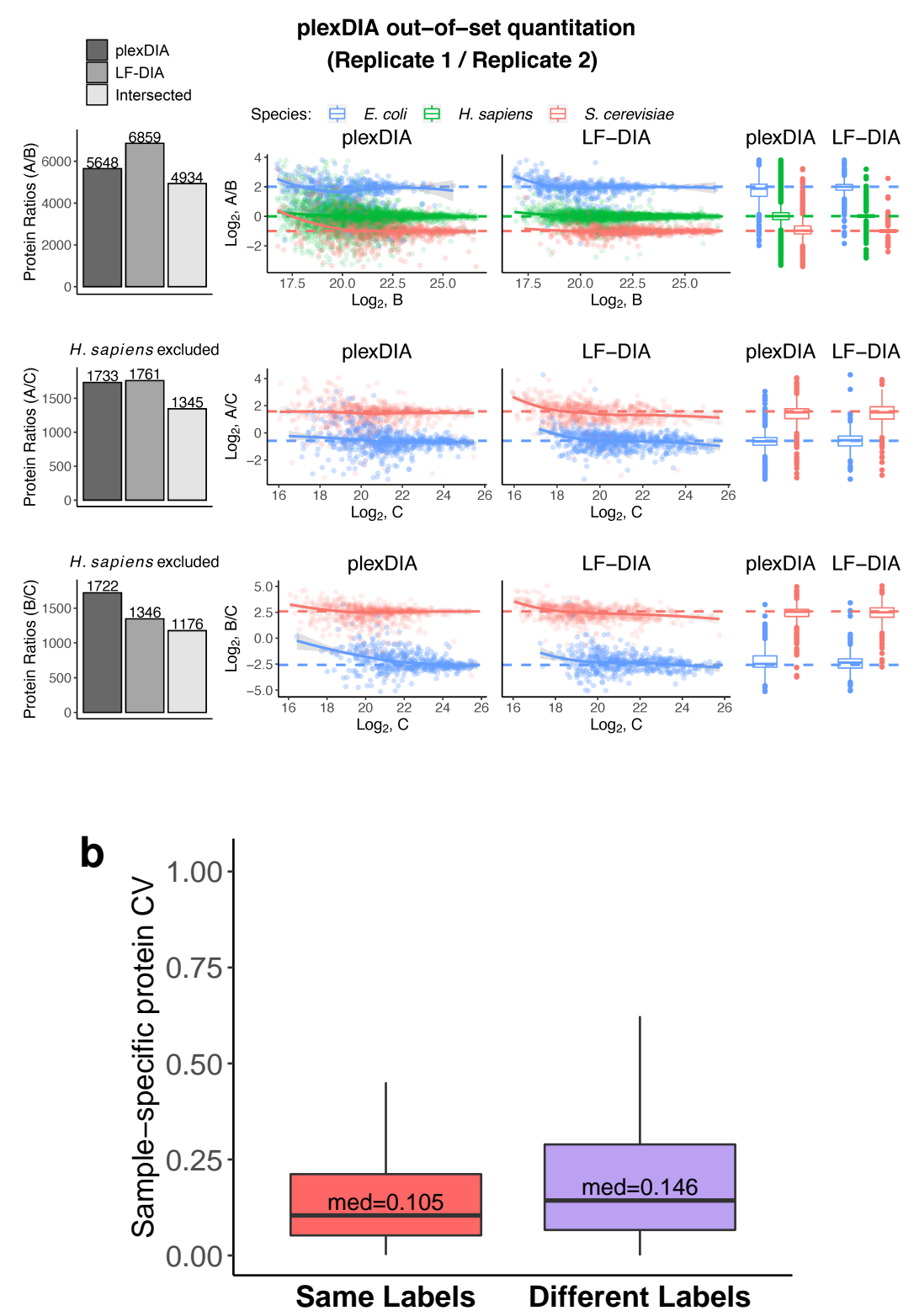

Figure S5 $\mid$ Quantitative accuracy and reproducibility across different plexDIA sets and labels. (a) Relative protein levels between samples A, B and C estimated from samples analyzed in different plexDIA sets, i.e. out-of-set quantification. The quantitative accuracy between sets (and thus runs) is comparable to the within set accuracy shown in Figure 3. The display is the same as shown in main Figure 3, but the protein ratios are estimated across runs (e.g. run $1 \mathrm{~A} /$ run $2 \mathrm{~B}$ ); LF-DIA is showing protein ratios for the 2nd replicate of samples A,B,C. (b) Quantitative reproducibility of plexDIA across across different labels. Protein CVs were estimated for the same samples labeled with the same label (as in main Fig. 4) or for the same sample labeled with different labels in different runs, e.g. run $1, \Delta 0$, sample A \& run $2, \Delta 4$, sample $A \&$ run $3, \Delta 8$, sample A. Both distributions contains CV for the same proteins, a set 15,199 sample-specific protein data points per condition (Same Labels or Different Labels). The median CV when using the same label was 0.105 while the label swap had a median CV of 0.146 

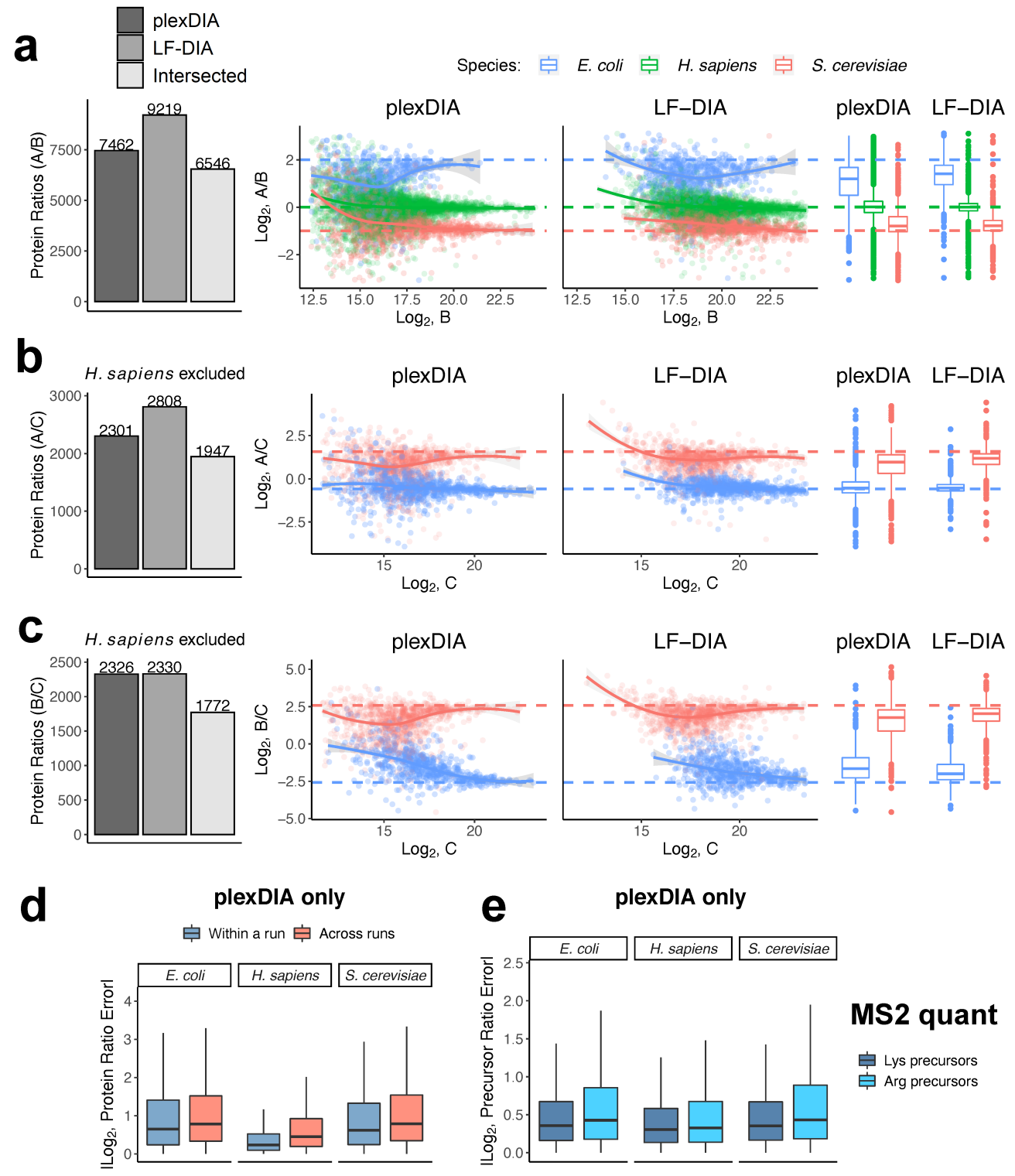

MS2 quant

Lys precursors

追 Arg precursors

Figure S6 | plexDIA quantitative accuracy for MS2-optimized data acquisition (V2) As demonstrated with the MS1-optimized method in Figure 3 of the main text, here we show quantitative accuracy of plexDIA using MS2-optimized data acquisition - specifically, we only show data from the second run of a triplicate set. (a) The number of protein groups quantified in both samples $A$ and $B$ is shown with barplots. plexDIA quantified 7,462 PGs, LF-DIA 9,219 PGs, and intersected between plexDIA and LF-DIA was 6,546 PGs. These 6,546 PGs were plotted to compare quantitative accuracy between plexDIA and LF-DIA for in-common protein groups. (b) Same as (a), but for samples A and C; human proteins were excluded because they compare two different human cell-types. (c) Same as (b), but for samples B and C. (d) Absolute protein ratio errors were calculated for samples $A / B, A / C$, and $B / C$ and combined to compare ratio errors for samples within a plexDIA run (e.g., run2 A / run2 B), to samples across runs (e.g., run1 A /run2 B) with plexDIA. (e) Absolute precursor ratio errors were calculated for samples $A / B, A / C$, and $B / C$ and combined to compare MS2-quantified ratio errors for $\mathrm{C}$-terminal lysine precursors and $\mathrm{C}$-terminal arginine precursors. 


\section{Relative protein abundance of each species per label}

\begin{tabular}{|l|l|l|l|}
\hline & $\Delta 0$ & $\Delta 4$ & $\Delta 8$ \\
\hline E. coli & $20 \%$ & $5 \%$ & $30 \%$ \\
\hline S. cerevisiae & $15 \%$ & $30 \%$ & $5 \%$ \\
\hline U-937 & $65 \%$ & $65 \%$ & $0 \%$ \\
\hline Jurkat & $0 \%$ & $0 \%$ & $65 \%$ \\
\hline
\end{tabular}

Table S1 | Relative protein abundances for each species per label Distribution of relative protein abundance of each species across labels. The pooled sample $\Delta 0, \Delta 4$, and $\Delta 8$ was used for quantitative benchmarking of plexDIA 